Aus der Abteilung Psychiatrie und Psychotherapie

(Prof. Dr. P. Falkai)

im Zentrum Psychologische Medizin

der Medizinischen Fakultät der Universität Göttingen

\title{
Zum Langzeitverlauf und zur Mortalität von Benzodiazepinabhängigen im Vergleich zu Kontrollen
}

\author{
INAUGURAL-DISSERTATION \\ zur Erlangung des Doktorgrades \\ der Medizinischen Fakultät \\ der Georg-August-Universität zu Göttingen
}

vorgelegt von

Esther Wißmüller

aus

Fürth

Göttingen 2012 
De k a n:

Prof. Dr. med. M. Schön

I. Berichterstatter: Prof. Dr. med. W. Poser

II. Berichterstatter/in: Prof. Dr. med. H. J. Steinfelder

III. Berichterstatter/in: Prof. Dr. rer. nat. P. Virsik-Köpp

Tag der mündlichen Prüfung: 29.10.2012 


\section{Inhaltsverzeichnis}

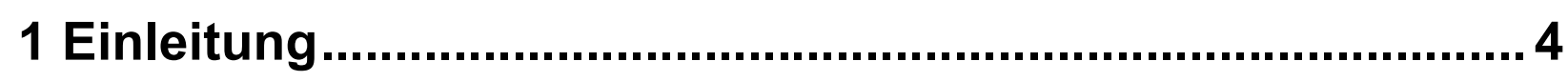

1.1 Zur Geschichte der Benzodiazepine als Arzneimittel ........................................4

1.2 Definitionen, Diagnostik und Formen der Benzodiazepin-abhängigkeit......................7

1.3 Die Mortalität benzodiazepinabhängiger Patienten im Literaturvergleich .....................11

2 Fragestellung 15

3 Patienten und Methoden .................................................... 16

3.1 Studienbeschreibung und Genehmigung durch die Ethik-Kommission ....................16

3.2 Zusammensetzung des Patientenkollektivs....................................................17

3.3 Untersuchungsmethodik und Datenschutz ……............................................19

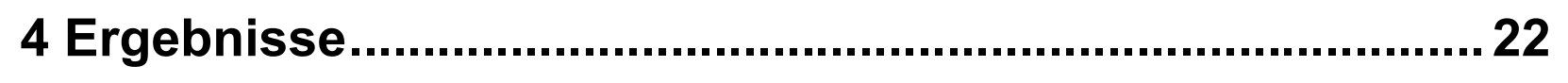

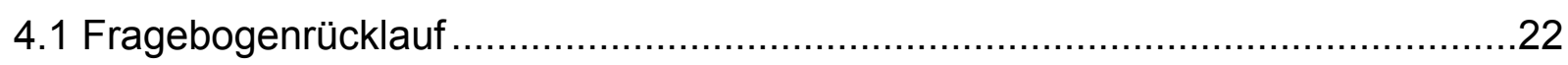

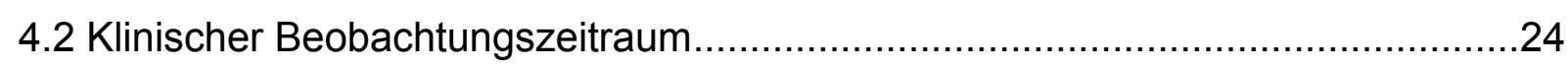

4.3 Verteilungstests von Alter und Beobachtungszeit...........................................25

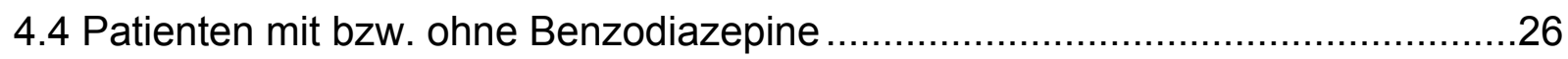

4.4.1 Vergleich der days and years under observation ....................................26

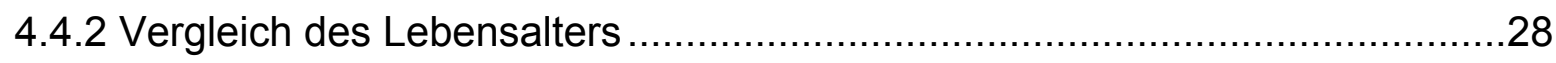

4.4.3 Geschlechtsverteilung (insgesamt 207 Patienten) ...................................29

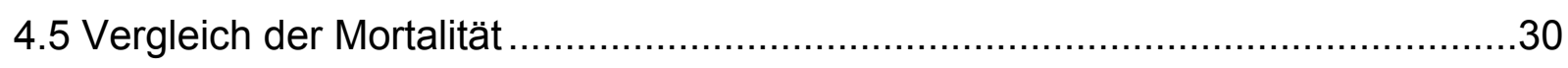

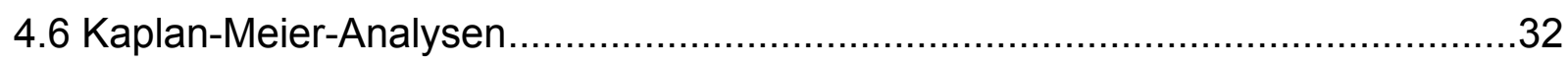

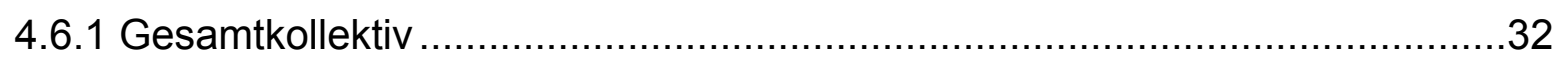

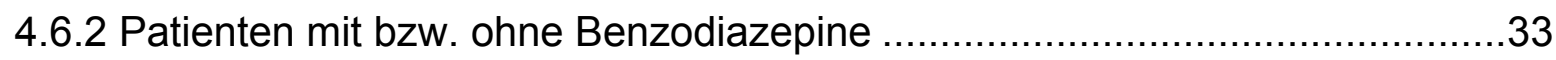

4.6.3 Mortalität in Abhängigkeit vom Geschlecht .............................................35

4.7 Anmerkungen zur Anwendung der statistischen Methoden..............................36

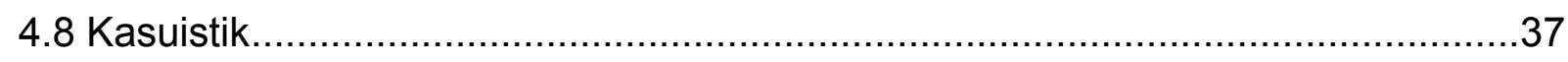




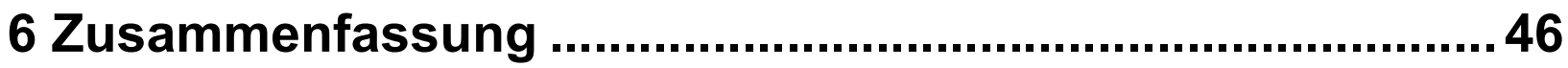

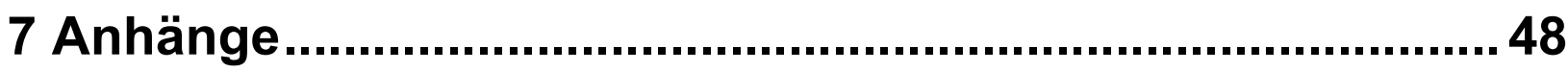

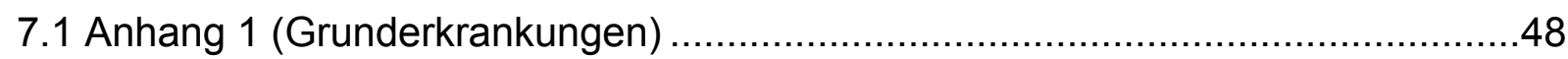

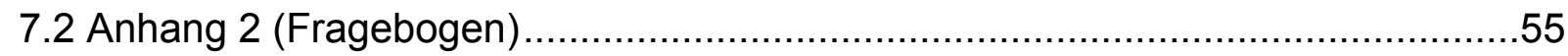

7.3 Anhang 3 (Ergebnisse der Fragebogen zum Langzeitverlauf) …........................58

7.4 Anhang 4 (Ergebnisse bezüglich des Entzuges) ...........................................69

7.5 Anhang 5 (Reaktionen der Patienten auf Einwilligung / Fragebogen) ..................70

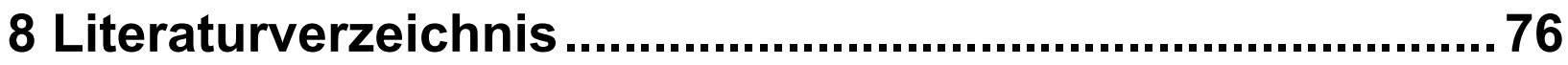

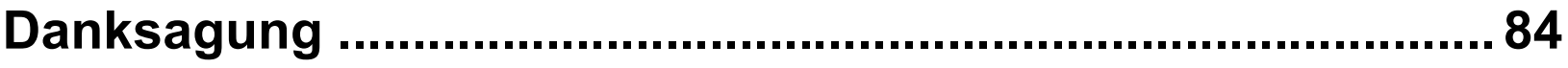

Lebenslauf .............................. Fehler! Textmarke nicht definiert. 


\section{Einleitung}

\subsection{Zur Geschichte der Benzodiazepine als Arzneimittel}

Die Geschichte der Benzodiazepine begann in Krakau (Polen) Mitte der 30erJahre des 20. Jahrhunderts (Sternbach 1983). Der Chemiker Dr. Leo Henryk Sternbach arbeitete dort mit einer chemischen Gruppe von Heterocyclen, den Benzoheptoxdiazinen, die sich später als Chinazolin-3-Oxide herausstellten (Linde 1988). Sternbach ging 1941 in die USA, wo er im Chemical Research Department von Hoffmann-La Roche in Nutley, New Jersey, u.a. zahlreiche Benzodiazepine synthetisierte. Dabei ging er von seinen alten Untersuchungen mit Benzoheptoxdiazinen/Chinazolin-3-Oxiden aus (Linde 1988).

Im April 1957 war im Labor von Sternbach eine unerträgliche Situation entstanden. Die Labortische waren übersät mit Schüsseln, Schalen, Flaschen und sonstigem Gerät. Eine vernünftige Laborarbeit war nicht mehr möglich. Deshalb wurde ein Großreinemachen angeordnet. Während dieser Arbeiten machte inn sein Mitarbeiter Earl Reeder auf wenige hundert Milligramm von zwei Substanzen aufmerksam, auf eine kristalline Base und das zugehörige Hydrochlorid. Diese waren bereits 1955 synthetisiert, aber noch keinen pharmakologischen Tests unterzogen worden. Erste Versuche mit dem wasserlöslichen Salz wurden erst 1957 unternommen. Die Ergebnisse waren zunächst unbrauchbar, was das Ende der Arbeit mit Chinazolin-3-Oxiden zu bedeuten schien. Randall, ein anderer Mitarbeiter Sternbachs, machte wenig später darauf aufmerksam, dass diese Verbindung in den sechs Tests, die gewöhnlich für die Voruntersuchungen für Tranquilizer und Sedativa durchgeführt wurden, ungewöhnlich interessante Eigenschaften hatten. Die weiteren Untersuchungen wurden von Randall durchgeführt. Diese zeigten, dass die Verbindung doch kein Chinazolin-N-Oxid war, sondern ein Benzodiazepinderivat, das anstelle eines 6-gliedrigen Pyrimidinringes einen 7-gliedrigen Diazepinring enthielt. Der Reaktionsmechanismus wurde aufgeklärt und in der Folge wurden weitere verwandte Verbindungen synthetisiert (Sternbach 1971). Deshalb wurde als 
Grundlage für weitere Untersuchungen das erste Benzodiazepinderivat, für das im Juli 1959 ein Patent ausgestellt wurde, gewählt. All diese Benzodiazepinderivate besaßen ähnliche biologische Eigenschaften. Zunächst war jedoch keines besser oder signifikant verschieden von der ersten Verbindung. Deshalb wurde als Grundlage für weitere Untersuchungen das erste Benzodiazepinderivat ausgewählt.

Langzeit-Toxizitätsstudien erbrachten ausgezeichnete Ergebnisse und auch die klinischen Untersuchungen schienen sehr vielversprechend. Das Interesse der klinischen Untersucher wuchs dermaßen, dass in relativ kurzer Zeit ungefähr 16.000 Patienten mit dem Medikament behandelt wurden. Schnell wurde die Zulassung durch die Food and Drug Administration erteilt. Unter dem Handelsnamen Librium wurde das neue Medikament 1960 eingeführt, nur zweieinhalb Jahre nach dem Beginn der pharmakologischen Untersuchungen. Der generic name, der später generell anerkannt wurde, war „Chlordiazepoxid“.

Im Verlauf wurden weitere Substanzen entwickelt, die sich aber im Wesentlichen nur in ihrer Rezeptoraffinität und damit in der wirksamen Dosis unterschieden. Obwohl es schwierig ist, aus tierexperimentellen Daten Voraussagen über den klinischen Effekt zentral wirkender Substanzen beim Menschen zu treffen, wurde damals die tierexperimentell wirksamste Verbindung (auf Gewichtsbasis) ausgewählt. Dieses Produkt mit dem internationalen Freinamen Diazepam kam 1963 unter dem Handelsnamen Valium auf den Markt. Es hatte ein breiteres Wirkspektrum als Librium. Besonders die muskelrelaxierenden Eigenschaften waren intensiv, die Toxizität im Vergleich zu anderen Benzodiazepinen sehr niedrig. Das Diazepam wurde zur Leitsubstanz der Benzodiazepinreihe.

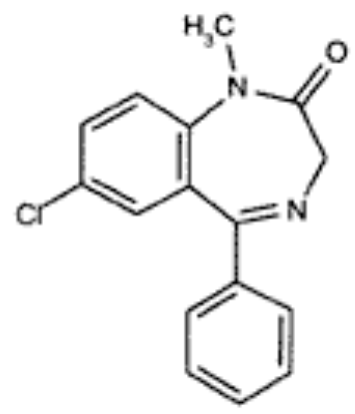

Diazepam: 7-Chlor- 1-methyl- 5-phenyl- 1,3-dihydro- 1,4-benzodiazepin-2-on $\mathrm{C}_{16} \mathrm{H}_{13} \mathrm{CIN}$ O (IUPAC) 
Im weiteren Verlauf wurden in wenigen Jahren über 3.000 1,4-Benzo- und Heterodiazepine synthetisiert. Eine große Anzahl 1,4-Benzodiazepinderivate wurde im Laufe der Jahre auf dem Arzneimittelmarkt eingeführt, was den klinischen Wert dieser Verbindungen zeigt. Im Jahre 1983 standen dem Kliniker in Deutschland über 25 Verbindungen zur Verfügung. Die meisten davon waren Anxiolytika, einige Hypnotika und ein Produkt (Clonazepam) wird speziell bei Epilepsie eingesetzt (Haefely 1986, Di Chiara und Imperato 1988, APA 1990). Später kam das wasserlösliche Midazolam hinzu, das heute ein wichtiges Kurznarkosemittel ist (Kanto 1985).

Der Wirkmechanismus der Benzodiazepine beruht auf der spezifischen Bindung an zentrale Benzodiazepinrezeptoren. Diese bewirkt eine verlängerte Öffnung von Chlorid-Kanälen und damit eine verstärkte Wirkung des dämpfenden Neurotransmitters GABA $=$ $ү$-Gamma-Aminobuttersäure (Facklam et al. 1992). Die Benzodiazepinwirkung ist von der GABA-Konzentration am Rezeptor abhängig. Sie kann theoretisch nicht stärker werden als die bei maximaler GABAKonzentration. Jedoch wird die einfache Dosis-Wirkungsbeziehung bzw. die Linksverschiebung der Dosis-Wirkungskurve durch die Existenz unterschiedlicher GABA-Rezeptorpopulationen kompliziert (Möhler et al. 2002), weil folglich eine Vielzahl an Kombinationen von GABA-Rezeptorkanalkomplexen möglich ist.

Nachdem der Einsatz von Benzodiazepinen in den 60er- und 70er-Jahren dramatisch zugenommen hatte, nahm der Gebrauch als Tranquilizer, aber nicht der als Hypnotika ab (Lader 1991). Im Laufe der Jahre haben Benzodiazepine eine weit verbreitete Anwendung gefunden (Ausweitung der Indikationen), die in manchen Bereichen weit über das vernünftige Maß hinausgeht. Gründe für die starke Verbreitung sind der schnelle Wirkungseintritt und die initial niedrige Nebenwirkungsrate. Zurzeit sind folgende Indikationen für Benzodiazepine und verwandte Substanzen in der Medizin akzeptiert:

- Schwere Angstzustände (einschließlich Panikattacken)

- Schwere Unruhezustände

- Kurznarkosen und Narkoseeinleitungen, auch als Kombinationspartner 
- Ein- und Durchschlafstörungen

- Epilepsietherapie (nur einige Formen, vor allem Initialbehandlung und Behandlung akuter Anfälle bzw. Status epilepticus)

- Behandlung schwerer Alkoholentzugssyndrome (Delirium tremens)

- Initialbehandlung von schweren und psychotischen Depressionen

- Katatone Schizophrenien

- Muskelverspannungen

- Stiff-man-Syndrom.

\subsection{Definitionen, Diagnostik und Formen der Benzodiazepin- abhängigkeit}

Eine erste Publikation zu Entzugserscheinungen nach längerfristiger Einnahme von Chlordiazepoxid erschien bereits 1961, nur ein Jahr nach der Markteinführung (Hollister et al. 1961). Der erste eindeutige Bericht über Abhängigkeitsentwicklungen nach Absetzen einer Benzodiazepin-Dauereinnahme erschien 1964 (Essig 1964). Lange wurden ein möglicher Abusus (heute: schädlicher Gebrauch) und eine mögliche Abhängigkeit von Benzodiazepinen vehement geleugnet, bis Zahlen Gegenteiliges bewiesen. Die allgemeine Überzeugung von Suchtfreiheit konnte wissenschaftlich nicht mehr gehalten werden (Keup 1993). Seit vielen Jahrzehnten ist die Benzodiazepinabhängigkeit die häufigste Arzneimittelabhängigkeit, nicht nur in Deutschland, sondern weltweit (Keup 1993, Wolf et al. 1994). Es kann davon ausgegangen werden, dass alle derzeit zur Verfügung stehenden Anxiolytika, Tranquilizer und Schlafmittel der Benzodiazepin-Reihe (einschließlich Thienozepine, Zaleplon, Zolpidem und Zopiclon) ein Abhängigkeitspotenzial besitzen und dass dieses Abhängigkeitspotenzial bei allen Substanzen vergleichbar groß ist. Es spielt allerdings bei der Wahl des missbrauchten Benzodiazepins eine größere Zahl von Faktoren eine Rolle: Der wichtigste Faktor ist die Verfügbarkeit, und diese wiederum ist sehr stark mitbestimmt vom Umsatz- und dem Verschreibungsvolumen der verschiedenen Benzodiazepine auf dem Markt bzw. in der praktischen Medizin (Keup 1993). 
Weiterhin spielen Anflutungsgeschwindigkeit, Eliminationshalbwertszeit und aktive Metabolite eine Rolle.

Viele Suchtkrankheiten entwickeln sich auf dem Boden einer vorbestehenden anderen Suchtkrankheit, z.B. einer Alkoholabhängigkeit. Dagegen entwickeln Patienten mit isolierter Benzodiazepinabhängigkeit diese meistens bei vorhergehenden anderen Erkrankungen, z.B. Angststörungen, BorderlinePersönlichkeitsstörungen oder Depressionen. Die lebenslang isolierte Benzodiazepinabhängigkeit ist deutlich seltener als die isolierte Abhängigkeit nach vorhergehender anderer Suchtkrankheit (Platz 1986).

Der Terminus Sucht ist seit der Völkerwanderung gemeingermanischer Herkunft und hat die Ursprungsbedeutung Krankheit (Grimmsches Wörterbuch, Grimm und Grimm 2004). Diese Bedeutung findet sich bis heute in Komposita wie Gelbsucht oder Bauchwassersucht. Der Wortstamm selbst ist in siech $=$ krank und in Sucht $=$ „Missbrauch und Abhängigkeit von Stoffen“ erhalten geblieben.

Im Roche Lexikon Medizin (2003, S. 1779) wird der Suchtbegriff so definiert:

„Sucht: unscharfer, umgangssprachl. Begriff, der einerseits den gesamten Komplex der Drogen- und Alkoholabhängigkeit umfasst, andererseits u.a. Spiel-, Ess- u. sexuelle Sucht einschließt. Sollte der World Health Organization zufolge durch Abhängigkeit ersetzt werden.“

Bemerkenswert ist, dass das von der Fa. Hoffmann-La Roche AG herausgegebene Lexikon die Arzneimittelabhängigkeit (= Medikamentenabhängigkeit) verschweigt: Hoffmann-La Roche war schließlich in den 60er Jahren der wichtigste Hersteller der abhängigkeitserzeugenden Benzodiazepine. 
Heute wird der Terminus Sucht in der offiziellen WHO-Nomenklatur ICD-10 (ICD10-WHO Version 2011) durch den Begriff „Psychische und Verhaltensstörungen durch psychotrope Substanzen“ ersetzt, der aber wegen seiner Länge noch gern als „Sucht“ abgekürzt wird. Er ist auf die substanzbedingten Störungen schädlicher Gebrauch = Abusus und Abhängigkeit beschränkt, wobei die „nichtstofflichen Süchte“, vor allem Glücksspielsucht um Geld und Bulimie manchmal eingeschlossen werden. Die Krankheitsdefinitionen von ICD-10 werden weltweit - die USA ausgenommen - verwendet, wo das DSM-IV TR (APA 2003) eingesetzt wird. DSM-IV TR ist dem ICD-10 ähnlich, jedoch in manchen Details spezifischer und besser handhabbar. Jedoch umfasst es nur die psychischen Krankheiten und wird in Deutschland von den Krankenkassen und dem Gesetzgeber nicht anerkannt.

In der neueren Literatur (Schmidt et al. 2006, S. 277) unterscheiden manche Autoren zwischen schädlichem Gebrauch (misuse), Missbrauch (abuse) und Abhängigkeit (dependence) und definieren die Begriffe wie folgt:

1. „Schädlicher Gebrauch“ (misuse) (nach ICD-10: F 13.1)

"bezeichnet ein Konsummuster von Sedativa oder Hypnotika, das zu einer physischen oder psychischen Gesundheitsschädigung führt (z.B. Unfälle). Der isolierte schädliche Gebrauch (ohne gleichzeitige Abhängigkeit) ist selten und führt noch seltener zum Behandlungswunsch; [...].“

Anmerkung der Autorin: Hier fehlen die sozialen Schäden, z.B. Inhaftierungen, Arbeitsplatzverluste und Partnerverluste.

\section{2. „Missbrauch“ (abuse)}

„Hingegen kommt Missbrauch (im Sinne von DSM-IV) von Sedativa/Hypnotika im Rahmen der Polytoxikomanie häufig vor, vor allem bei Abhängigen von illegalen Drogen."

Unter Polytoxikomanie wird die Abhängigkeit von mehreren Suchtmitteln verstanden, und zwar gleichzeitig oder nacheinander (multiple drug dependence). Der Missbrauch mehrerer Suchtstoffe gleichzeitig wird als „polyvalenter Missbrauch" bezeichnet. 
Immer muss unterschieden werden, ob die Stoffe gleichzeitig oder nacheinander genommen wurden. Dies ist für die Beurteilung der einzelnen Untersuchungen wichtig.

\section{3. „Abhängigkeit“ (dependence)}

„wird im Sinne des ,Abhängigkeitssyndroms“ (ICD-10 der Weltgesundheitsorganisation [2000]) bzw. der Substanzabhängigkeit (Diagnostische Kriterien DSM-IV der American Psychiatric Association [2003]) in beiden Klassifikationssystemen praktisch identisch verwandt [...]."

Alle Fachleute sind sich einig, dass bei Benzodiazepinsuchten die Abhängigkeit bei weitem überwiegt, während der schädliche Gebrauch selten ist (Wolf et al. 1994, Poser W und Poser S 1996, Lader 2011). Meist sind "schädlicher Gebrauch“ und „Abusus“ Fehldiagnosen, hinter denen sich eine Abhängigkeit verbirgt.

Wie Poser W et al. (2006) in: „Medikamentenabhängigkeit (Sedativa, Hypnotika, Analgetika, Psychostimulanzien)" in Tabelle 7.2 (S. 278) zeigen, besteht zwischen ICD-10 und DSM-IV noch immer keine völlige Übereinstimmung, was zu Missverständnissen und unterschiedlichen Definitionen führen kann. So taucht das Wort „addiction“ noch immer in der englischsprachigen Literatur auf. O'Brien (2005) stellt fest, dass „Sucht” im DSM-IV „Abhängigkeit“ genannt wird. Trotzdem beschreibt er den Begriff „addiction“ ausführlich und kommt zu dem Fazit, dass, obwohl physische Abhängigkeit für die Sucht typisch ist, die physiologische Abhängigkeit kein erforderliches Kriterium für Sucht ist. De las Cuevas et al. (2003, S. 301) schreiben: „Obwohl das Wort, ,Abhängigkeit‘ wissenschaftlich generell mehr anerkannt ist, sollte das Wort ,Sucht' bevorzugt werden, da es die Natur der Benzodiazepin-Gebrauchsprobleme genauer wiedergeben könnte."

Damit ist klar, dass in der Suchtforschung und in der klinischen Psychiatrie immer noch keine vollständige Einigkeit über die Definition der Begriffe „Abhängigkeit“ und "Sucht" sowie die dahinterstehenden Krankheitsbilder besteht. 


\subsection{Die Mortalität benzodiazepinabhängiger Patienten im Literaturvergleich}

Wie bei allen Suchtkrankheiten, liegt auch bei der isolierten Benzodiazepinabhängigkeit eine gegenüber der Normalbevölkerung erhöhte Sterblichkeitsrate vor (Piesiur-Strehlow et al. 1986, Poser W et al. 1992, Hausken et al. 2007, Belleville 2010). Über die Mortalität von Patienten, die von „legalen Drogen“ abhängig (heute besser „Arzneimittelabhängige“) sind, war damals wenig bekannt. Zwar wurde eine gegenüber der Normalbevölkerung (besser: Gesamtbevölkerung) leicht erhöhte Mortalität gefunden, es wurde aber nicht untersucht, ob die Mortalitätserhöhung eher durch die Benzodiazepinabhängigkeit oder eher durch die Grunderkrankung(en) verursacht wird. Die typischen Grunderkrankungen (z.B. Depressionen, Angststörungen und BorderlinePersönlichkeitsstörungen) von Benzodiazepinabhängigen haben per se eine gegenüber der Gesamtbevölkerung erhöhte Mortalität (Kawachi et al. 1996, Celano und Huffmann 2011, Smoller et al. 2007, van't Veer-Tazelaar et al. 2006). Bei bestimmten Populationen (z.B. über 65-Jährige) kann die Mortalität von Konsumenten, Nichtabhängigen, sogar der Normalbevölkerung entsprechen, wenn nach Alter, Geschlecht, Antipsychotikagebrauch und Diagnosen adjustiert wird (Gisev et al. 2011). Das gilt in abgeschwächter Form auch für Menschen mittleren Alters (Hausken et al. 2007). Letztere weisen zwar eine erhöhte Mortalität im Vergleich zur Gesamtbevölkerung auf, die Differenz verkleinert sich aber, wenn konfundierende Variablen (u.a. Schmerzmittelgebrauch, Zigarettenkonsum) kontrolliert werden.

Die langfristige Abstinenzrate (länger als 1 Jahr) nach erfolgtem ambulanten oder stationären Entzug wurde bisher in der Fachliteratur wenig untersucht. Immerhin liegen einige Publikationen vor, die für eine recht hohe Abstinenzrate sprechen (de Gier et al. 2011, Zitman und Couvée 2011). Allerdings sind die optimalen Interventionen für dieses Therapieziel unklar (Vicens et al. 2011). Es scheinen sogar relativ geringfügige Interventionen auszureichen („minimal intervention“, de Gier et al. 2011). Vor allem aber fehlen Untersuchungen zum Einfluss der Abstinenz auf die Mortalität von Benzodiazepinabhängigen. 
Die vorliegende Arbeit beschäftigt sich mit dem Mortalitätsvergleich von Patienten mit isolierter Benzodiazepinabhängigkeit und deren Kontrollpatienten, die nicht abhängig sind, aber an der gleichen Grunderkrankung leiden. Deshalb wurde diese Follow-up-Studie für die damals (und heute) häufigste Medikamentenabhängigkeit in der deutschen Bevölkerung durchgeführt. Ein besonderes Augenmerk wurde auf isoliert Benzodiazepinabhängige gelegt. In den Jahren 1972 bis 1982 wurden in der Klinik für Psychiatrie und Psychotherapie der Universität Göttingen mehr als 400 Fälle von Benzodiazepinabhängigkeit untersucht. Dabei handelte es sich jedoch vornehmlich um Patienten, die einen oder mehrere zusätzliche Suchtstoffe nahmen (Alkohol, illegale Drogen oder legale Arzneimittel). Nur eine Minderzahl der Patienten wies eine lebenslange isolierte Benzodiazepinabhängigkeit auf. Von 387 eingeschlossenen Patienten waren nur 68 isoliert über die gesamte bisherige Lebensdauer benzodiazepinabhängig.

Als Datengrundlage wurden die Krankengeschichten der Klinik sowie an die Patienten versandte Fragebogen verwendet. Berichte anderer Krankenhäuser wurden nur verwendet, wenn sie in der Krankengeschichte der Psychiatrischen Universitätsklinik Göttingen enthalten waren. Weiterhin wurden allfällige Todesdaten bzw. aktuelle Adressen von den kommunalen Einwohnermeldeämtern erfragt.

Patienten mit einer isolierten Benzodiazepinabhängigkeit wiesen gegenüber der Durchschnittspopulation eine erhöhte Sterblichkeitsrate auf. Die Mortalität unterschied sich jedoch nicht von der Mortalität der Kontrollgruppe, die auch in dieser Studie in Geschlecht, Geburtsdatum und in der vorbestehenden psychiatrischen Erkrankung übereinstimmte. Diese Studie ergab zum damaligen Zeitpunkt keinen eindeutigen Beweis eines erhöhten Risikos für eine erhöhte Mortalität, die auf eine isolierte Benzodiazepinabhängigkeit zurückzuführen war. Es wurde jedoch die bekannte Tatsache bestätigt, dass beachtlich erhöhte Mortalitätsraten bei solchen Abhängigen bestanden, die Benzodiazepine mit Alkohol und/oder anderen Arzneimitteln bzw. Drogen kombinierten. 
1984 wurde eine zweite Follow-up-Studie mit einer angestiegenen „time under risk", die sich nur auf die isolierte Benzodiazepinabhängigkeit beschränkte, durchgeführt. In 64 eingeschlossenen Fällen wurde auch die Methode der "matched pairs“ angewendet. Für jeden benzodiazepinabhängigen Patienten wurde ein nicht benzodiazepinabhängiger „Partner“ möglichst übereinstimmend in Geschlecht, Alter und psychiatrischer Erkrankung ausgesucht.

Letztendlich konnte auch hier sowohl bei den isoliert Benzodiazepinabhängigen als auch in der Gruppe der Nichtabhängigen nachgewiesen werden, dass die Mortalität im Vergleich zur Durchschnittspopulation erhöht war. Die Mortalitäten beider Gruppen unterschieden sich nicht. Dies lässt vermuten, dass die leicht erhöhte Mortalität beider Gruppen auf die vorbestehende psychiatrische Erkrankung zurückzuführen war.

Für die Gruppe der isoliert benzodiazepinabhängigen Patienten konnte also nur eine leichte Tendenz einer erhöhten Mortalität ausgemacht werden. Diese erreichte jedoch keine statistische Signifikanz. Das Ergebnis hängt aber vermutlich mit der geringen Zahl der untersuchten Fälle und den wenigen Jahren unter Beobachtung zusammen.

Auch in weiteren Arbeiten wurde eine erhöhte Mortalität unter Suchtkranken im Vergleich zur Normalbevölkerung angegeben. Eine von Poser W et al. durchgeführte Auswertung eines seit 1974 geführten Fallregisters der Klinik („Suchtkatamnese Südniedersachsen“) ergab eine massiv erhöhte Sterblichkeit besonders beim Missbrauch von Arzneimitteln plus illegaler Drogen (17-fach). Bei einem Abusus und einer Abhängigkeit von Arzneimitteln allein stieg die Mortalität weniger massiv an (etwa 2-fach). Hier ist die Übersterblichkeit zwar gering, wenn auch vorhanden und nachweisbar, und die Prognose hinsichtlich Sterblichkeit und Abstinenz ist bei dieser Patientengruppe günstiger. Abusus war von der Sterblichkeit her gesehen weniger bedrohlich als Abhängigkeit. Allerdings war schädlicher Gebrauch (früher „Abusus“) seltener als Abhängigkeit. In dieser Arbeit wurde im Hinblick auf die Todesursachen auch festgestellt, dass bei allen Suchtkranken häufig unnatürliche Todesarten vorkamen. 
1974 wurde von Poser W et al. eine Follow-up-Studie über Patienten mit unterschiedlichen Suchtkrankheiten, amerikanisch „psychoactive substance use disorders (PSUD)“ begonnen (Strehlow et al. 1988). Wieder wurde eine erhöhte Mortalität in allen Gruppen mit „PSUD“ nachgewiesen.

In einer Arbeit von 1999 (Rintahaka et al.) wurde beschrieben, dass die erhobenen Daten einen Zusammenhang zwischen Nitrazepameinnahme und einer erhöhten Mortalität bei Kindern mit „intractable epilepsy“ anzeigen. Die aufgetretenen Todesfälle in der Benzodiazepingruppe zeigten eine höhere Mortalität im Vergleich zur Patientengruppe ohne Benzodiazepine.

In einer weiteren Studie wurden prospektiv die Folgen der Verschreibung von Benzodiazepinen bei älteren (85 Jahre und mehr) Niederländern (Vinkers et al. 2003) beschrieben: Es kam gehäuft zu schlimmen Ereignissen wie Stürzen, Verkehrsunfällen, etc. Aber es blieb unklar, in welchem Ausmaß der ausgedehnte Gebrauch von Benzodiazepinen einen Einfluss auf das Mortalitätsrisiko hat, da o.g. Unfälle in der genannten Altersgruppe auch ohne Benzodiazepinkonsum häufig vorkommen. Das Mortalitätsrisiko für Patienten mit Benzodiazepineinnahme verglichen mit denen ohne Benzodiazepingebrauch war nicht sicher erhöht.

In allen oben genannten Publikationen werden kaum Fälle von isolierter Benzodiazepinabhängigkeit, sondern solche mit Benzodiazepinkonsum oder kombinierter Abhängigkeit (meist Alkohol plus Benzodiazepine) beschrieben. 2003 erschien eine Studie (Poser W und Wegerer 2001) mit der Beobachtung, dass eine Benzodiazepinabhängigkeit eine geringere Übersterblichkeit und bessere Abstinenzchancen hat als z.B. eine Alkoholabhängigkeit. Hierbei wurden die aktuell isoliert Benzodiazepinabhängigen betrachtet. Der Langzeitverlauf wurde nicht systematisch untersucht, z.B. die Frage des „Umsteigens“ (Wechsel von einem Suchtstoff zum anderen) oder der Polytoxikomanie. Vor allem fehlte der Vergleich zwischen nichtabhängigen Benzodiazepinkonsumenten mit Benzodiazepinabhängigen. 
Deshalb soll in der vorliegenden Arbeit die Sterblichkeit der isoliert benzodiazepinabhängigen Patienten im Kontext von Behandlungs- und Beobachtungsjahren untersucht werden.

\section{Fragestellung}

Nach der Fachliteratur ist die Mortalität benzodiazepinabhängiger Patienten im Vergleich zur Normalbevölkerung erhöht. Die Mehrzahl dieser Patienten leidet jedoch neben der Abhängigkeit an weiteren psychischen oder körperlichen Erkrankungen (Komorbidität), die ebenfalls mit erhöhter Mortalität einhergehen. Es bleibt somit offen, ob die Abhängigkeit oder die komorbide(n) Störung(en) Ursache der erhöhten Mortalität ist (sind). Deshalb soll in dieser Arbeit untersucht werden, ob die Mortalität nur bei Abhängigen oder auch bei einer parallelisierten Kontrollgruppe mit vergleichbarer Alters-, Geschlechts- und Krankheitsstruktur, aber ohne Abhängigkeit, erhöht ist.

Weiterhin sollen die Hospitalisierungsdauer und die Nachbeobachtungsdauer („years under observation“) der Patientengruppen verglichen werden. War ein Patient mit einer Benzodiazepinabhängigkeit länger stationär oder ambulant in der Klinik in Behandlung als ein Patient mit der gleichen Grunderkrankung, jedoch ohne Abhängigkeitsleiden?

Schließlich wird untersucht, ob ein wesentlicher Altersunterschied bei Stellung der Erstdiagnose besteht. Wie alt sind die Patienten mit isolierter Benzodiazepinabhängigkeit bei Aufnahme in die Klinik? In welchem Lebensalter tritt eine isolierte Benzodiazepinabhängigkeit auf? Die Geschlechtsverteilung wird ebenfalls betrachtet. 


\section{Patienten und Methoden}

\subsection{Studienbeschreibung und Genehmigung durch die Ethik- Kommission}

In der vorliegenden Fall-Kontroll-Studie sollte die Mortalität einer benzodiazepinabhängigen Patientengruppe mit der einer nichtabhängigen Kontrollgruppe verglichen werden. Die Kontrollgruppe sollte dabei ein vergleichbares Krankheitsspektrum aufweisen, weil die große Mehrzahl der Benzodiazepinabhängigen an psychischen, seltener neurologischen Vorerkrankungen leidet. Diese Primärerkrankungen hatten durch die regelmäßige Benzodiazepinmedikation den Ausgangspunkt für die Abhängigkeitsentwicklung gebildet.

Da der Langzeitverlauf bei Benzodiazepinabhängigkeit (im Gegensatz zum Benzodiazepinkonsum) bisher noch nicht ausreichend untersucht wurde, liegt der Schwerpunkt dieser Studie auf der Beobachtung der abhängigen Patienten über den stationären oder ambulanten Beobachtungszeitraum hinaus.

Das Studiendesign berücksichtigt u.a. die deutschen Datenschutzgesetze und die Deklaration von Helsinki (World Medical Association 2000, 5. Revision). Im Mai 2006 wurde der Ethik-Kommission der Georg-August-Universität Göttingen eine Studienskizze unter der Antragsnummer 15/5/06 vorgelegt. Am 22.11.2006 teilte die Ethik-Kommission mit, dass keine ethischen und rechtlichen Bedenken bezüglich des vorgelegten Studienvorhabens bestünden. Nachdem dieses positive Votum der Ethik-Kommission der Medizinischen Fakultät Göttingen vorlag, wurde mit der Studie begonnen. 


\subsection{Zusammensetzung des Patientenkollektivs}

Es wurde eine Vollerhebung aller bisher erfassten isoliert benzodiazepinabhängigen Patienten der Göttinger Universitätsklinik mit Bildung einer parallelisierten Kontrollgruppe durchgeführt. Dieses Vorgehen lehnt sich an das Vorgehen von Allgulander (1978) für schwedische Patienten an, mit zwei gravierenden Unterschieden:

- Bei Allgulander wurde die Kontrollstichprobe aus der schwedischen Normalbevölkerung gezogen und war somit mehrheitlich gesund.

- Die Studiengruppe von Allgulander umfasste nicht nur Benzodiazepinabhängige, sondern auch Abhängige von anderen Sedativa/Hypnotika, z.B. solche von Barbituraten.

Ausgangspunkt sind alle früheren und aktuellen Patienten der Klinik für Psychiatrie und Psychotherapie der Universität Göttingen mit isolierter Benzodiazepinabhängigkeit. Diese waren im Rahmen der klinikeigenen DiagnoseDokumentation erfasst. Zum Zeitpunkt dieser Studie lagen rund 300 Krankengeschichten von Benzodiazepinabhängigen aus den Jahren 1961 bis 2007 vor.

\section{Beobachtungszeitraum der Studie:}

- Erfassung der Erstdiagnosedaten vom 28.12.1961 bis 16.11.2005 (44 Jahre)

- Erfassung der Katamnesedaten vom 08.10.1976 bis 05.09.2007 (31 Jahre).

Einschlusskriterien waren die isolierte Benzodiazepinabhängigkeit bzw. keine Einnahme von Benzodiazepinen (=Kontrollgruppe) und eine vorliegende Einwilligung der Patienten nach Aufklärung. Patienten mit einem Lebensalter unter 16 oder über 90 Jahren wurden bei der Erstdiagnose nicht beobachtet. 
Zunächst wurden die Krankengeschichten von benzodiazepinabhängigen Patienten studiert, um die Art der Benzodiazepinabhängigkeit (isoliert oder kombiniert mit anderen Suchtstoffen) festzulegen. Patienten mit kombinierter Abhängigkeit zum Erstdiagnosezeitpunkt wurden ausgeschlossen. Dann wurden daraus die Parallelisierungsparameter extrahiert, um aus diesem Unterkollektiv die Kontrollgruppe zu bilden.

Die Kontrollgruppe wurde mit Patienten der Klinik gebildet, die nie abhängig waren. Sie sollten mit jeweils einem abhängigen Patienten nicht nur in Geschlecht, Geburtsdatum (+/- 10 Jahre), Eintrittsalter in die Klinik (+/- 10 Jahre), sondern auch in der vorausgehenden psychiatrischen oder somatischen Grunderkrankung übereinstimmen. Kontrollpatienten, die nach der Krankengeschichte später eine Benzodiazepinabhängigkeit entwickelt hatten (nach Erstaufnahme), wurden ausgeschlossen. Bei den Patienten mit Benzodiazepinabhängigkeit bedeutet Grunderkrankung die Erkrankung, die zur Verschreibung eines Benzodiazepins führte. Außerdem wurden die Nebendiagnosen sowie vorausgegangene, aber bei Studienbeginn beendete Abhängigkeiten erfasst (siehe Anhang 1).

Es wurde darauf geachtet, dass der angesetzte, relativ weite Zeitraum von $+/-10$ Jahren nur in Ausnahmefällen herangezogen wurde. Deshalb wurde versucht, den jeweils am besten geeigneten Kontrollpatienten zu finden. Wenn der abhängige Patient und seine Kontrolle sowohl hinsichtlich Geburtsdatum als auch Eintrittsalter in die Klinik nicht übereinstimmten, wurde nur das Geburtsdatum berücksichtigt. Jedem Entzugspatienten wurde ein Kontrollpatient nach der "method of matched pairs" zugeordnet. Wenn kein Kontrollpatient auffindbar war, wurde der Patient nicht in die Studie eingeschlossen. Dies war nur bei wenigen Patienten der Fall. In einem Fall musste bei der Parallelisierung der Grunderkrankung von der strengen Parallelität abgewichen werden. Der Grunderkrankung "Torticollis spasmodicus“ (beim Benzodiazepinabhängigen) wurde deshalb eine Kontrolle mit der Grunderkrankung „bipolare Störung“ zugewiesen. 
Bei zwölf Benzodiazepinabhängigen waren aus den jeweiligen Patientenakten die jeweiligen Grunderkrankungen nicht eindeutig zu ermitteln. Hier wurden zum Teil ihre psychiatrischen Nebendiagnosen zur Auswahl der jeweiligen Kontrolle oder ein Kontrollpatient mit der Diagnose „Depression“ („depressive Episode“ oder "neurotische Depression = Dysthymie“) herangezogen .

Insgesamt wurden 207 isoliert benzodiazepinabhängige Patienten und ihre jeweiligen Kontrollpatienten in die Studie einbezogen. Somit wurden in dieser Studie insgesamt 414 Fälle betrachtet.

Tabelle 3.1: Krankenkollektiv

\begin{tabular}{|c|c|c|}
\hline & Häufigkeit & Prozent \\
\hline Kontrolle & 207 & 50,0 \\
\hline Benzodiazepine & 207 & 50,0 \\
\hline Gesamt & 414 & 100,0 \\
\hline
\end{tabular}

\subsection{Untersuchungsmethodik und Datenschutz}

Alle Benzodiazepinabhängigen wurden postalisch per Fragebogen nachbefragt. Im Vorfeld wurde innen ein Anschreiben, welches die Ziele der Untersuchung sowie eine ausführliche Beschreibung des gesamten Ablaufes der Befragung erläutert, zugeschickt. Auf einem Antwortschreiben konnten die angeschriebenen Personen erklären, ob sie zur Teilnahme bereit seien oder nicht. Dieses Anschreiben wurde den Patienten in zweifacher Ausfertigung zugesandt. Eines der beiden Exemplare konnten sie unterschrieben mit inrer jeweiligen Antwort (Zusage oder Absage zur Teilnahme) in einem vorfrankierten Rückumschlag zurücksenden. Bei bestehender rechtlicher Betreuung (Personensorge) wurde um zusätzliches Einverständnis des Betreuers gebeten. Dies war jedoch bei keinem Patienten der Fall.

Bei Nichtantwort wurde der Anfragebogen erneut übersendet. Dies betraf 75 Patienten. 
Wenn trotz zweimaligem Anschreiben keine Reaktion des ehemaligen Patienten erfolgte, wurde beim jeweils zuständigen Einwohnermeldeamt der aktuelle Wohnsitz des entsprechenden Patienten erfragt. Auf diese Weise konnte entweder eine neue Adresse des Patienten ermittelt werden oder es wurde die Auskunft erteilt, dass der Patient verstorben sei. Eine Vielzahl von Patienten sind seit ihrer Behandlung in der Abteilung Psychiatrie und Psychotherapie mehrmals umgezogen. Somit mussten in vielen Fällen verschiedene Einwohnermeldeämter angeschrieben werden, um aktuelle Informationen über den Verbleib ehemaliger Patienten zu erhalten. In einigen Fällen war es nicht möglich den Aufenthaltsort zu erfahren, da auch einige Einwohnermeldeämter über einen aktuellen Wohnsitz nicht informiert waren.

Teilte ein Patient auf das Anfrageschreiben mit, dass er an der Befragung teilnehmen wolle, so erfolgte in einem zweiten Schritt die Zusendung des pseudonymisierten Fragebogens (siehe Fragebogen im Anhang 2). Aufgrund der Tatsache, dass die Mehrzahl der Patienten einen der Untersucher (Poser W) persönlich kannte, gingen wir davon aus, dass die Rücklaufquote hoch sein würde (in einer früheren und vergleichbaren Untersuchung betrug diese über $80 \%$ ).

Solange es der Stand der Datenerfassung erlaubte, konnten die Patienten ihre Einwilligung zur Teilnahme widerrufen. Davon wurde jedoch kein Gebrauch gemacht. Die Kontrollpatienten wurden nicht per Fragebogen befragt. Hier lag das Interesse nur auf der Tatsache, ob sie noch lebten oder bereits verstorben waren. Allerdings wurde anhand ihrer Krankengeschichte überprüft, ob sich Hinweise für die Entwicklung einer Suchtkrankheit für den Zeitpunkt nach deren Berücksichtigung in die vorliegende Studie ergaben. Diese Methode ist relativ effektiv, weil die Mehrzahl der Kontrollpatienten (ebenso wie die Benzodiazepinabhängigen) erneut stationär aufgenommen worden war.

Das Auffinden der Akte eines Patienten, die schriftliche Voranfrage und die Rücklaufquote der positiven oder negativen Antworten wurden registriert. Die Rücklaufquote der pseudonymisierten Fragebogen nach erteilter Einwilligung wurde ebenfalls dokumentiert, ebenso die Auskünfte der Einwohnermeldeämter. 
Die relevanten Parameter der benzodiazepinabhängigen Patienten waren: Geschlecht, Geburtsdatum, Erstdiagnosedatum, Katamnesedatum, Alter bei Erstdiagnose, Beobachtungstage (days under observation), auslösende Grunderkrankung, vorhergehende Suchten, Art des Benzodiazepins, Datum der ersten Einnahme, Dosissteigerung, Alter bei Suchtbeginn, Nebendiagnosen, Zusatzmedikation, lebend oder verstorben, erfolgter Entzug und Art der Entzugssymptome. Die Parameter der Kontrollpatienten waren: Geschlecht, Geburtsdatum, Erstdiagnosedatum, Katamnesedatum, Alter bei Erstdiagnose, lebend oder verstorben, Grunderkrankung und Beobachtungstage (days under observation). Die Angaben in den anonymisierten Fragebogen und Auskünfte der Einwohnermeldeämter wurden in eine Excel-Tabelle eingegeben und dort ausgewertet. Der Entzugserfolg (siehe Anhang 4) sowie Mitteilungen der Patienten oder durch Angehörige per Telefonat, Brief oder E-Mail (nur Benzodiazepinabhängige) wurden deskriptiv erfasst (siehe Anhang 5).

Die Daten wurden auf einem PC ohne jegliche Netzanbindung im speziell gesicherten Archiv der Klinik für Psychiatrie und Psychotherapie erfasst und kontrolliert. Gesichert wurden diese auf einem USB-Stick, der in einem Safe in einem anderen Raum der Klinik aufbewahrt wurde. Die Zuverlässigkeit der Eingabe wurde durch Doppeleingabe, Zweitsicht und Plausibilitätsprüfungen festgestellt. Die Zuverlässigkeit der Fragebogenangaben wurde durch den Vergleich der Antworten des Fragebogens mit den Daten der Krankengeschichte überprüft. In Zweifelsfällen wurden die Angaben der Krankengeschichte verwendet.

Nach Rücklauf der Einwilligungsbestätigungen wurden die Namen, Geburtsdaten und Krankengeschichtsnummern durch eine Codenummer ersetzt (Pseudonymisierung). Die Schlüsselliste sowie die Einwilligung der Patienten wurden ebenfalls im speziell gesicherten Archiv der Abteilung gesondert aufbewahrt. Die Auswertungen erfolgten unter dem Pseudonym auf einem geeigneten Computer (s.o.) der Abteilung. Nach abgeschlossener Auswertung und Überprüfung auf Datenintegrität wurden die Codenummern auf der Festplatte und dem USB-Stick gelöscht (Anonymisierung) und die Schlüsselliste vernichtet. 
Das primäre Zielkriterium, die Gesamtmortalität der beiden Gruppen, wurde mittels Survival-Methoden (Kaplan-Meier-Kurve) ermittelt.

\section{Ergebnisse}

\subsection{Fragebogenrücklauf}

Insgesamt wurden 207 isoliert benzodiazepinabhängige Patienten in die Studie aufgenommen. 23 Personen waren laut Akten schon verstorben, somit konnten 184 Personen angeschrieben werden.

57-mal mussten Einverständniserklärungen, teils mehrfach (bis zu 4-mal) an Personen versendet werden, da diese Patienten mehrmals den Wohnort gewechselt hatten. Erst nach Anschreiben der jeweiligen Einwohnermeldeämter konnte in diesen Fällen die aktuelle Adresse ermittelt werden.

78 verschiedene Einwohnermeldeämter in ganz Deutschland wurden angeschrieben. Zudem wurde in einem Fall in Portugal nach einem dorthin verzogenen Patienten gesucht. Dies leider erfolglos, da Portugal kein dem deutschen Meldewesen vergleichbares System hat. Bei 78 Meldestellen wurde nach dem aktuellen Wohnsitz von 68 Patienten gefragt.

Von den 184 angeschriebenen Personen sandten 81 ihre Einverständniserklärungen zurück $\quad(44 \%)$. Davon wurden 34 Einverständniserklärungen mit „nein“ beantwortet. Das heißt, diese Patienten wollten an der Befragung mittels Fragebogen nicht teilnehmen (42\%). 47 Patienten erklärten sich einverstanden, sodass insgesamt 47 Fragebogen verschickt werden konnten (Fragebogen zum Langzeitverlauf siehe Anhang 2). Davon kamen insgesamt 43 beantwortete Fragebogen wieder zurück. Dies entspricht einer Rücklaufquote von $92 \%$. 
Somit beantworteten insgesamt 43 Patienten (23\%) der 184 angeschriebenen Patienten des benzodiazepinabhängigen Kollektivs den zugesandten Fragebogen (die zusammengefassten Antworten der erhaltenen 43 Fragebogen siehe Anhang 3). Von 24 Patienten (13\%) liegen Aussagen in Form von Anrufen, EMails oder schriftlichen Vermerken auf den nicht ausgefüllten Fragebogen vor (siehe Anhang 4).

Letztendlich können hiermit Aussagen über 128 Patienten (62\%) gemacht werden. Von 79 Patienten liegen keine Daten vor, da sie entweder unbekannt verzogen waren oder sich nicht auf unser Anschreiben meldeten (38 \%).

Tabelle 4.1: Nachuntersuchung

\begin{tabular}{|c|c|c|}
\hline & Anzahl & Prozent \\
\hline Laut Akte bereits verstorben & 23 & 11 \\
\hline Keine Teilnahme & 34 & 16 \\
\hline Anruf, E-Mail oder Vermerk & 24 & 12 \\
\hline Fragebogen beantwortet & 43 & 21 \\
\hline Fragebogen nicht beantwortet & 4 & 2 \\
\hline Unbekannt verzogen & 79 & 38 \\
\hline Gesamt & 207 & 100 \\
\hline
\end{tabular}

Das Studium der Krankengeschichten der Kontrollgruppe und der Benzodiazepingruppe wurde nahezu zeitgleich abgeschlossen. In der Kontrollgruppe wurden nur die Krankengeschichten ausgewertet, Fragebogen wurden nicht versandt. Die Nachuntersuchung der Benzodiazepingruppe erstreckte sich über einen Zeitraum von 11 Monaten (Anfang Mai 2006 bis Ende April 2007), wobei das mehrmalige und somit auch zeitaufwändige Anschreiben verschiedener Einwohnermeldeämter zu berücksichtigen ist. 


\subsection{Klinischer Beobachtungszeitraum}

Die Erstdiagnosedaten entstammen der Zeit vom 28.12.1961 bis zum 16.11.2005 und die der Katamnesedaten der Zeit vom 08.10.1976 bis zum 05.09.2007.

Im Durchschnitt wurden die Patienten beider Kollektive rund sieben Jahre nachbeobachtet. Für diesen Zeitraum standen dokumentierte Patientendaten zur Verfügung (Tabelle 4.2).

In der Gruppe der Benzodiazepinabhängigen mit 207 Patienten werden 1.217,16 Behandlungsjahre (Patientenjahre) erreicht. Das entspricht 5,88 Jahren pro Patient. Jedoch gab es drei Patienten, die nur für einen Tag beobachtet wurden.

Tabelle 4.2: Time under observation

\begin{tabular}{|l|l|c|c|}
\hline \multicolumn{2}{|l|}{} & $\begin{array}{c}\text { Days under } \\
\text { observation }\end{array}$ & $\begin{array}{c}\text { Years under } \\
\text { observation }\end{array}$ \\
\hline N & 414 & 414 \\
\hline Mittelwert & 2.576 & 7,061 \\
\hline Median & 1.365 & 3,700 \\
\hline Standardabweichung & 3.031 & 8,305 \\
\hline Maximum & 14.380 & 39,400 \\
\hline Perzentile & 25 & 95 &, 300 \\
\hline & 50 & 1.365 & 3,700 \\
\hline & 75 & 4.144 & 11,333 \\
\hline
\end{tabular}




\subsection{Verteilungstests von Alter und Beobachtungszeit}

Normalverteilungstests überprüfen die Übereinstimmung stetiger Zahlenreihen einer Stichprobe mit der Normalverteilung. Der Kolmogorov-Smirnov-Test wird angewendet, um zu prüfen, ob eine Zahlenreihe signifikant von der Normalverteilung abweicht. Eine signifikante Abweichung von der Normalverteilung wird angenommen, wenn die Zufallswahrscheinlichkeit unter $5 \%$ liegt $(p<0,05)$. In diesem Falle sind für die Signifikanztests der betreffenden Variablen nichtparametrische Tests zu benutzen.

Bei der Analyse der Ergebnisse zeigt sich, dass die Beobachtungszeiten (days and years under observation) mit einer Normalverteilung nicht vereinbar sind $(p<0,05$, Tabellen 4.3 und 4.4). Das Lebensalter zum Erstdiagnosezeitpunkt dagegen widerspricht einer Normalverteilung nicht ( $p>0,05$, Tabelle 4.5).

Tabelle 4.3: Jahre unter Beobachtung bei Patienten mit bzw. ohne Benzodiazepine

\begin{tabular}{|l|c|c|c|c|c|}
\hline & Mittelwert & $\begin{array}{c}\text { Standard- } \\
\text { Standard- } \\
\text { fehler des } \\
\text { abweichung }\end{array}$ & $\begin{array}{c}\text { Mittel- } \\
\text { wertes }\end{array}$ & Median & $\mathrm{N}$ \\
\hline Kontrolle & 8,239 & 8,777 &, 610 & 5,500 & 207 \\
\hline Benzodiazepine & 5,882 & 7,647 &, 531 & 1,720 & 207 \\
\hline Insgesamt & 7,061 & 8,305 &, 408 & 3,700 & 414 \\
\hline
\end{tabular}

Der Mann-Whitney-Test („Mann-Whitney-U-Test“ oder kurz „U-Test“) ist ein nichtparametrischer statistischer Test. Er wird anstelle des t-Tests bei Nichtvorliegen einer Normalverteilung eingesetzt. 
Tabelle 4.4: Mann-Whitney-U-Test für die Nachbeobachtungsdauer

\begin{tabular}{|l|c|c|}
\hline & $\begin{array}{c}\text { Days under } \\
\text { observation }\end{array}$ & $\begin{array}{c}\text { Years under } \\
\text { observation }\end{array}$ \\
\hline Z & $-3,062$ & $-3,113$ \\
\hline $\begin{array}{l}\text { Asymptotische } \\
\text { Signifikanz (2-seitig) }\end{array}$ &, 002 &, 002 \\
\hline
\end{tabular}

Somit ist die Kontrollgruppe im Mittel signifikant länger nachbeobachtet worden (Mann-Whitney-U-Test; $p=0,002$ ).

Tabelle 4.5: Tests auf Normalverteilung

\begin{tabular}{|l|c|c|c|}
\hline & \multicolumn{3}{|c|}{ Kolmogorov-Smirnov } \\
\hline & Statistik & df & Signifikanz \\
\hline $\begin{array}{l}\text { Alter bei } \\
\text { Erstdiagnose }\end{array}$ &, 042 & 414 &, 082 \\
\hline $\begin{array}{l}\text { Days under } \\
\text { observation }\end{array}$ &, 198 & 414 &, 000 \\
\hline $\begin{array}{l}\text { Years under } \\
\text { observation }\end{array}$ &, 198 & 414 &, 000 \\
\hline
\end{tabular}

Signifikanzkorrektur nach Lilliefors

\subsection{Patienten mit bzw. ohne Benzodiazepine}

\subsubsection{Vergleich der days and years under observation}

Bei den folgenden Mittelwertvergleichen der vorliegenden Stichproben der beiden Kollektive liegen unabhängige Stichproben vor; das heißt, dass die Werte in keinem direkten Zusammenhang stehen (Tabellen 4.6 und 4.7). Im Gegensatz dazu haben mehrfache Messungen in einem Kollektiv, z.B. vor und nach Therapie, 
eine direkte Verbindung. Bei unabhängigen und verbundenen Stichproben kommen unterschiedliche Testverfahren zum Einsatz.

Beim Vergleich von 2 unabhängigen, normalverteilten Stichproben wird der t-Test verwendet, dagegen beim Vergleich von 2 unabhängigen, nicht normalverteilten Stichproben der Mann-Whitney-U-Test.

Tabelle 4.6: Days under observation bei Patienten mit bzw. ohne Benzodiazepine

\begin{tabular}{|c|c|c|c|c|c|}
\hline Gruppe & Mittelwert & $\begin{array}{l}\text { Standard- } \\
\text { abweichung }\end{array}$ & $\begin{array}{c}\text { Standard- } \\
\text { fehler des } \\
\text { Mittel- } \\
\text { wertes }\end{array}$ & Median & $\mathrm{N}$ \\
\hline Kontrolle & 3.006 & 3.202 & 223 & 2.020 & 207 \\
\hline Benzodiazepine & 2.146 & 2.791 & 194 & 628 & 207 \\
\hline Insgesamt & 2.576 & 3.031 & 149 & 1.365 & 414 \\
\hline
\end{tabular}

Wie bereits erwähnt, standen die Patienten aus beiden Gruppen im Schnitt 7 Jahre in Kontakt mit der Klinik, die Patienten aus der Kontrollgruppe im Durchschnitt jedoch 2,3566 Jahre länger als die benzodiazepinabhängigen Patienten (Tabelle 4.7).

Tabelle 4.7: Years under observation bei Patienten mit bzw. ohne Benzodiazepine

\begin{tabular}{|c|c|c|c|c|c|}
\hline Gruppe & Mittelwert & $\begin{array}{l}\text { Standard- } \\
\text { abweichung }\end{array}$ & $\begin{array}{c}\text { Standard- } \\
\text { fehler des } \\
\text { Mittel- } \\
\text { wertes }\end{array}$ & Median & $\mathrm{N}$ \\
\hline Kontrolle & 8,239 & 8,777 & 610 & 5,500 & 207 \\
\hline Benzodiazepine & 5,882 & 7,647 & ,531 & 1,720 & 207 \\
\hline Insgesamt & 7,061 & 8,305 & ,408 & 3,700 & 414 \\
\hline
\end{tabular}




\subsubsection{Vergleich des Lebensalters}

Zwischen den beiden Gruppen besteht kein wesentlicher Unterschied hinsichtlich des Lebensalters bei Erstdiagnose. Auch ein statistisch bedeutsamer Geschlechtsunterschied ist nicht festzustellen.

Durchschnittsalter (Benzodiazepingruppe): 207 Patienten bei Erstdiagnose

Durchschnittsalter des Gesamtkollektivs : $\quad$ 48,2 Jahre

Durchschnittsalter der Frauen: $\quad$ 48,5 Jahre

Durchschnittsalter der Männer: 47,6 Jahre

Durchschnittsalter (Kontrollen): 207 Patienten bei Erstdiagnose

Durchschnittsalter des Gesamtkollektivs : $\quad$ 48,9 Jahre

Durchschnittsalter der Frauen: $\quad$ 49,8 Jahre

Durchschnittsalter der Männer: 47,2 Jahre

Die Verteilung des Lebensalters bei Erstaufnahme in die Klinik folgt der Gauss'schen Verteilungskurve.

Diagramm 4.1: Altersverteilung gesamt $(n=414)$

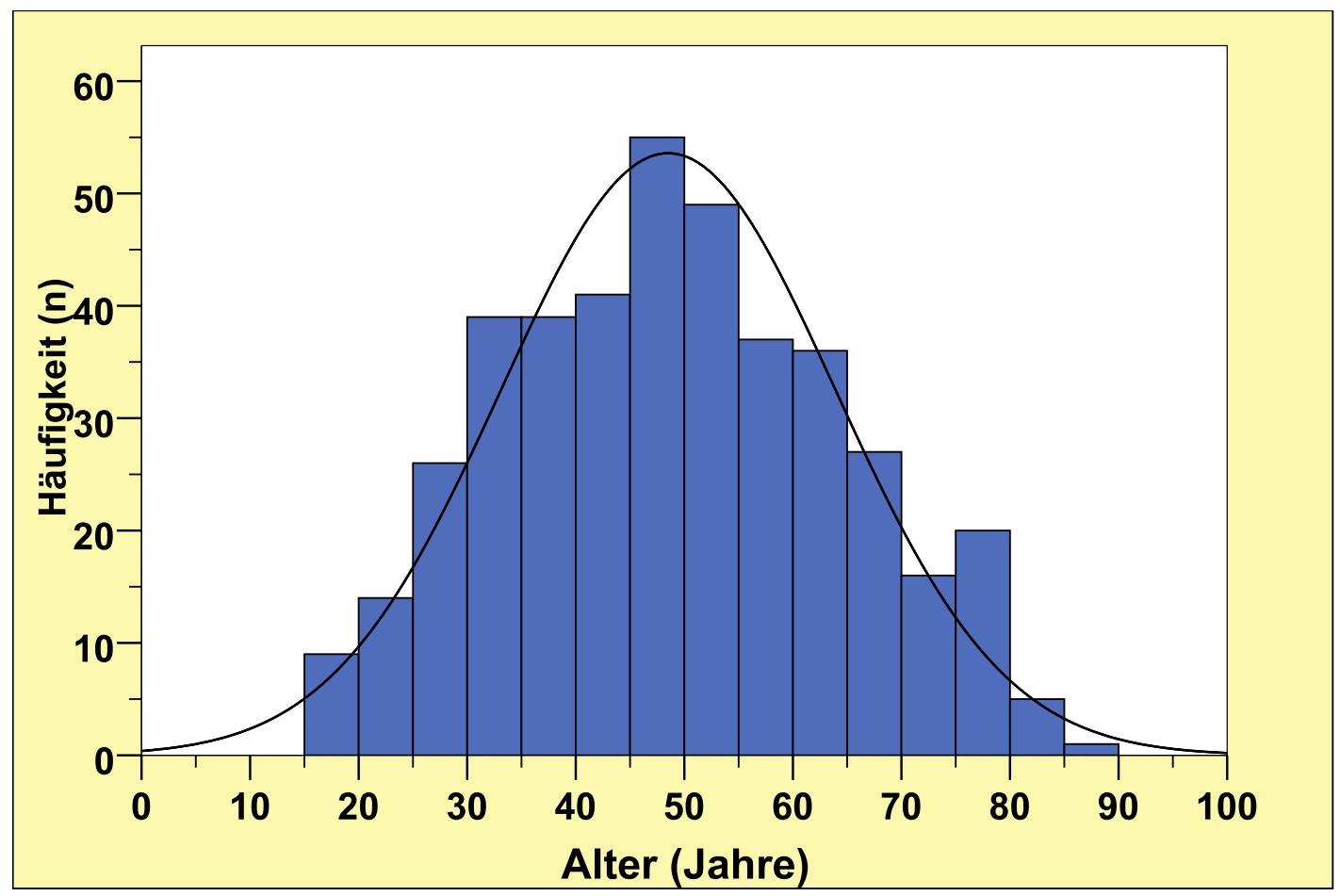


In der Kontrollgruppe $(\mathrm{n}=207)$ waren zum Zeitpunkt der Erstdiagnose der jüngste Patient 17 Jahre und die jüngste Patientin 16 Jahre alt. Der älteste Patient hatte ein Alter von 76 Jahren, die älteste Patientin war 85 Jahre alt. In der Gruppe der Benzodiazepinabhängigen waren zum Zeitpunkt der Erstdiagnose der jüngste Patient 18 Jahre und die jüngste Patientin 19 Jahre alt. Der älteste Patient hatte ein Alter von 83 Jahren, die älteste Patientin war 84 Jahre alt.

Diagramm 4.2: Alter bei Patienten mit bzw. ohne Benzodiazepine

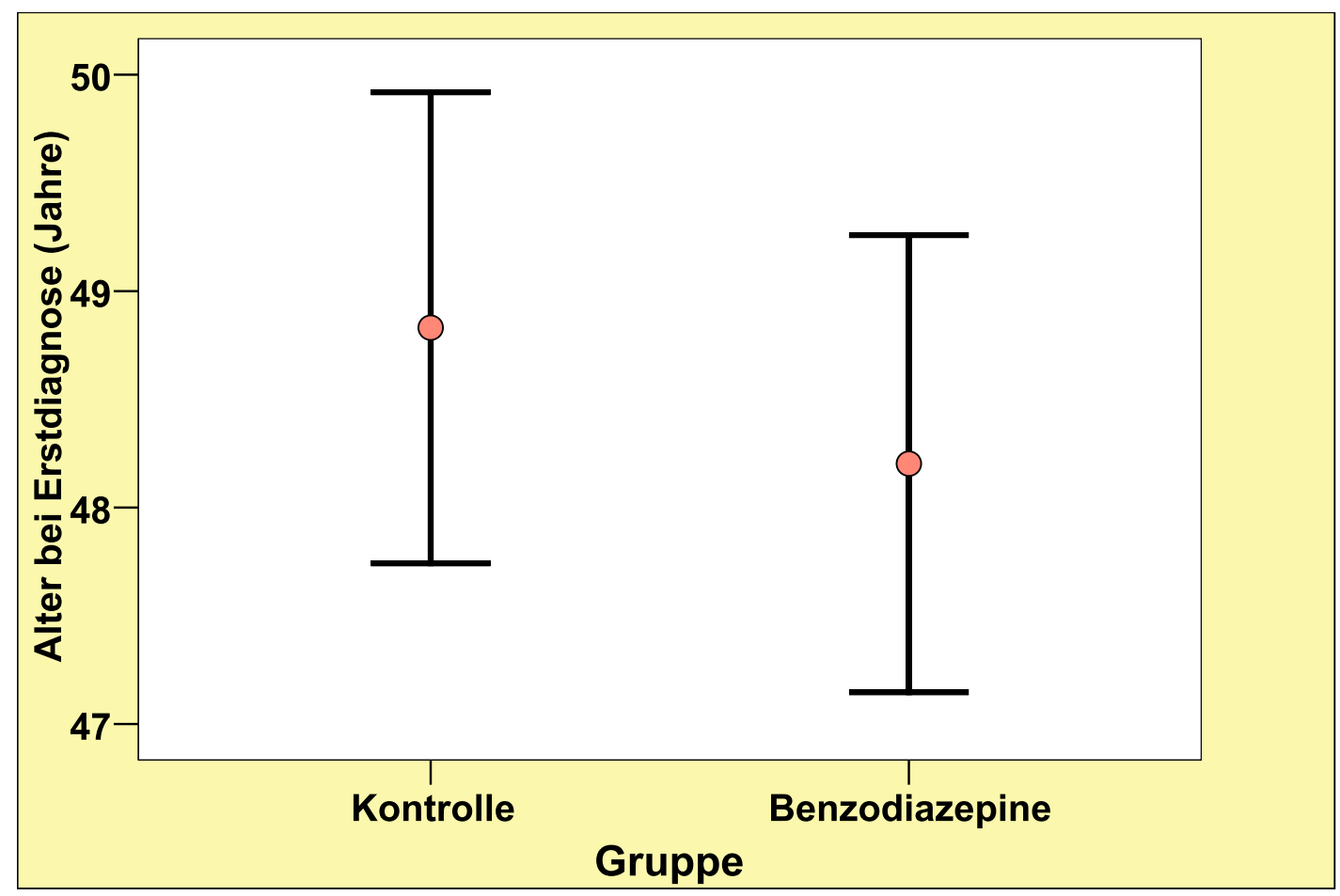

\subsubsection{Geschlechtsverteilung (insgesamt 207 Patienten)}

In beiden Kollektiven fanden sich jeweils 136 Frauen $=65,4 \%$ und 71 Männer $=$ $34,6 \%$, wobei naturgemäß kein Geschlechtsunterschied zwischen den Kollektiven bestand, da u.a. auf das Geschlecht parallelisiert wurde. In dieser Studie waren Frauen annähernd doppelt so häufig wie Männer vertreten (Diagramm 4.3). Das heißt, dass Frauen unter allen der bisher erfassten isoliert benzodiazepinabhängigen Patienten der Klinik für Psychiatrie und Psychotherapie der Universität Göttingen doppelt so häufig betroffen waren wie Männer 
(Diagramm 4.3). Dieses Faktum ist aus allen Untersuchungen zur Benzodiazepinabhängigkeit bekannt: Frauen sind deutlich häufiger betroffen (Piesiur-Strehlow et al. 1986, Vicens et al. 2011).

Diagramm 4.3: Geschlecht bei Patienten mit bzw. ohne Benzodiazepine

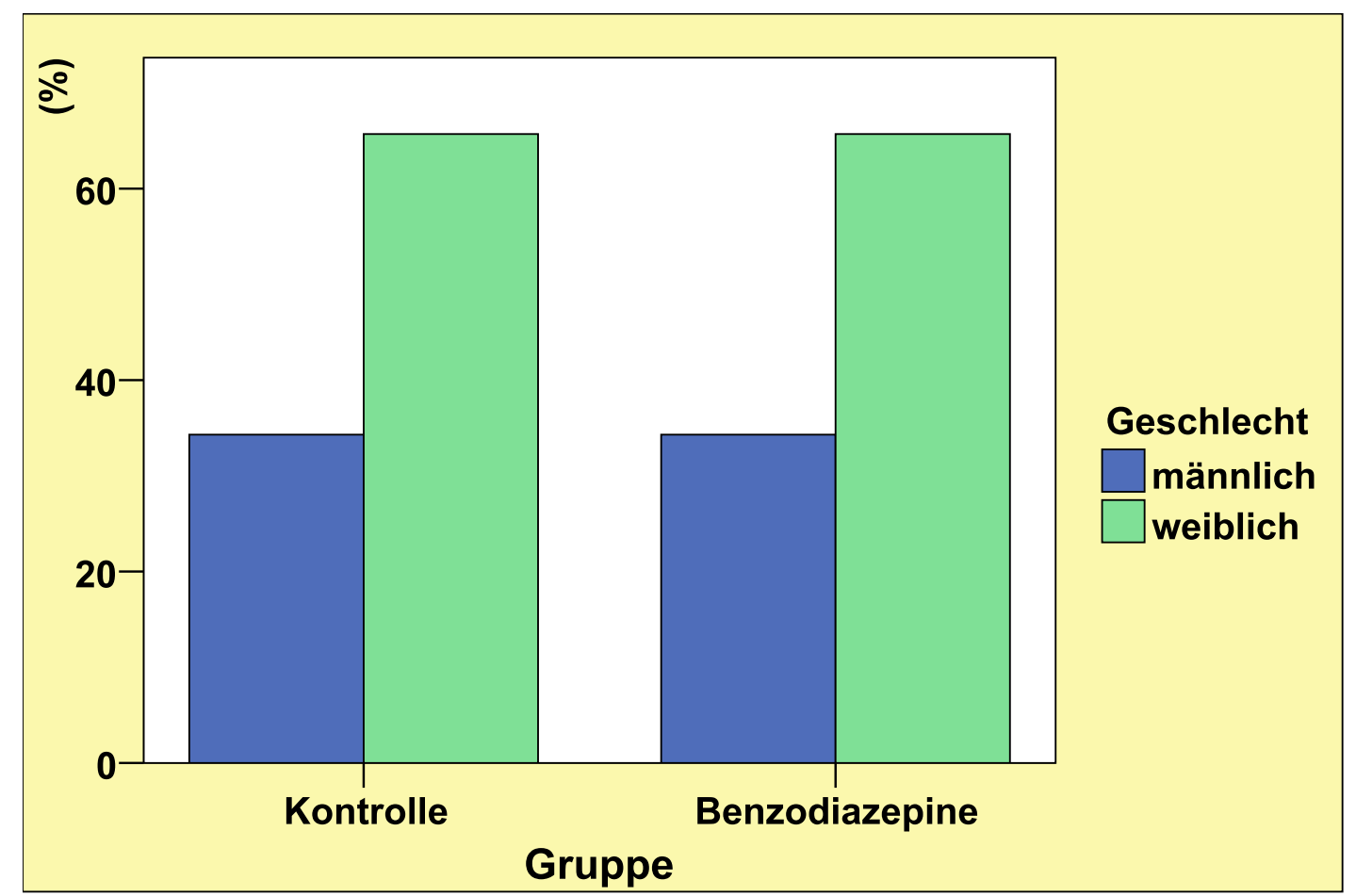

\subsection{Vergleich der Mortalität}

Von insgesamt 414 Patienten aus beiden Gruppen sind 89 Patienten, 21,5\%, verstorben (Tabelle 4.8).

In der Kontrollgruppe verstarben von 207 Patienten 32 Patienten (15,5\%). Bezogen auf alle verstorbenen Patienten aus beiden Gruppen (benzodiazepinabhängige Patienten und Kontrollpatienten) ist dies ein Anteil von $36,0 \%$. 
In der Benzodiazepingruppe verstarben von 207 Patienten insgesamt 57 Patienten (27,5\%). In Bezug auf das Gesamtkollektiv der Verstorbenen in dieser Studie beträgt die Anzahl der Verstorbenen aus dieser Gruppe 64,0 \% (Tabelle 4.8 und Diagramm 4.4).

Tabelle 4.8: Mortalität mit bzw. ohne Benzodiazepine

\begin{tabular}{|c|c|c|c|c|c|}
\hline & & \multicolumn{2}{|c|}{ verstorben } & \multirow[t]{2}{*}{ Summe } \\
\hline & & & nein & ja & \\
\hline \multirow[t]{6}{*}{ Gruppe } & Kontrolle & Anzahl & 175 & 32 & 207 \\
\hline & & $\%$ & $84,5 \%$ & $15,5 \%$ & $100 \%$ \\
\hline & Benzodiazepine & Anzahl & 150 & 57 & 207 \\
\hline & & $\%$ & $72,5 \%$ & $27,5 \%$ & $100 \%$ \\
\hline & Gesamt & Anzahl & 325 & 89 & 414 \\
\hline & & $\%$ & $78,5 \%$ & $21,5 \%$ & $100 \%$ \\
\hline
\end{tabular}

Diagramm 4.4: Mortalität mit bzw. ohne Benzodiazepine

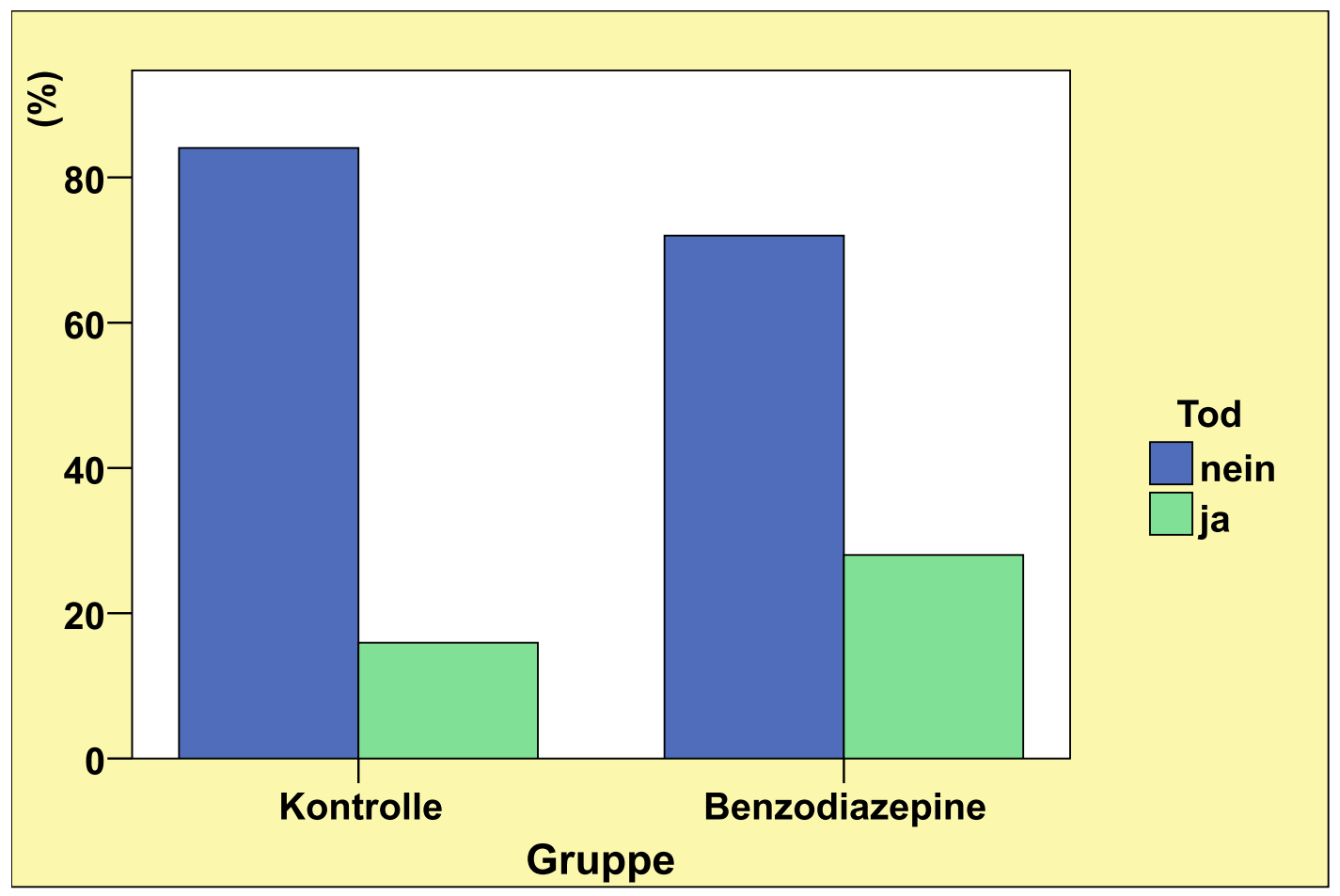




\subsection{Kaplan-Meier-Analysen}

\subsubsection{Gesamtkollektiv}

Die Überlebensraten können als Kaplan-Meier-Kurve dargestellt werden. Jede Stufe kennzeichnet die Abnahme der Patientenzahl durch Tod (Diagramm 4.5). Für jedes Intervall (i) kann die Wahrscheinlichkeit des Überlebens $\left(p_{i}\right)$ berechnet werden (Tabelle 4.9, 4.10).

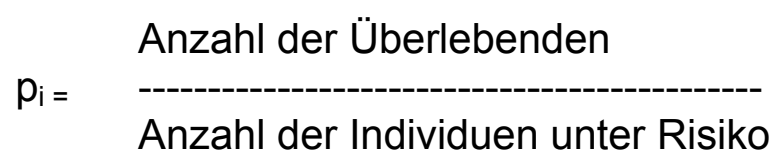

In der Regel wird die Kaplan-Meier-Kurve als Mittelwert mit einem $95 \%$ Konfidenzgürtel angegeben. Die Kaplan-Meier-Kurve einer Gruppe von Kranken mit einer bestimmten Krankheit, hier Benzodiazepinabhängige mit einer Grunderkrankung, kann mit einer nach Alter und Geschlecht entsprechenden Gruppe von Nichtbenzodiazepinabhängigen mit der gleichen Grunderkrankung verglichen und der Unterschied mit statistischen Verfahren auf Signifikanz geprüft werden. Daraus wird erkennbar, ob und in welchem Ausmaß sich der Krankheitsverlauf unter Abhängigkeit von Benzodiazepinen hinsichtlich der Lebenserwartung von der Lebenserwartung der Nichtbenzodiazepinabhängigen mit der gleichen Grunderkrankung unterscheidet (Gerok et al. 2007).

Diagramm 4.5: Gesamtmortalität

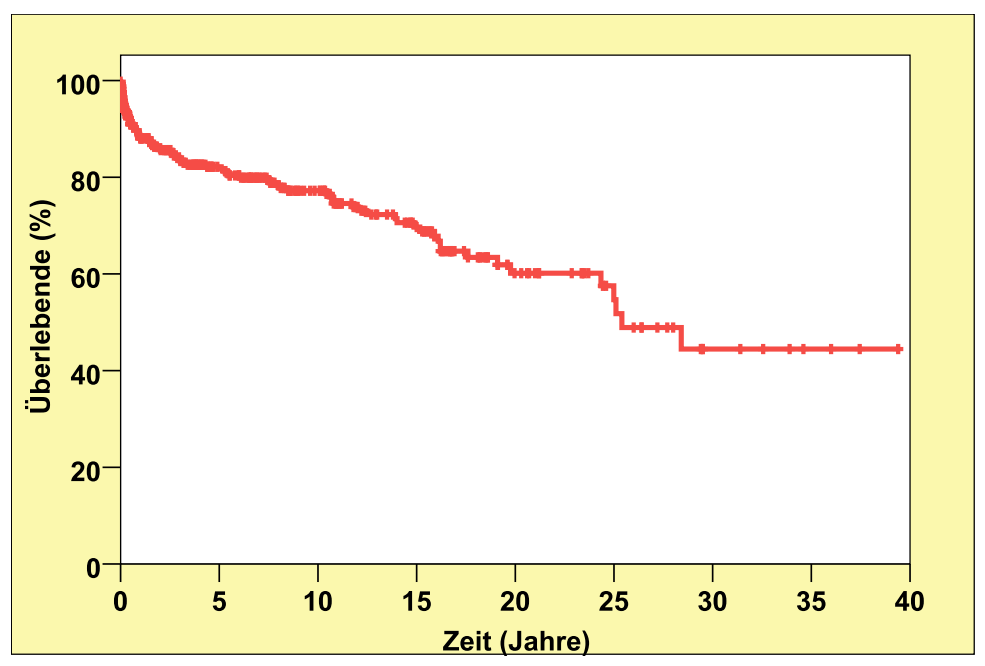


Tabelle 4.9: Gesamtmortalität

\begin{tabular}{|c|c|c|c|}
\hline $\begin{array}{c}\text { Gesamt- } \\
\text { zahl }\end{array}$ & $\begin{array}{c}\text { Anzahl der } \\
\text { Ereignisse }\end{array}$ & \multicolumn{2}{|c|}{ Zensiert } \\
\hline $\mathrm{N}$ & Prozent & $\mathrm{N}$ & Prozent \\
\hline 414 & 91 & 323 & $78,0 \%$ \\
\hline
\end{tabular}

Zensiert = lebt zum Nachuntersuchungszeitpunkt

Tabelle 4.10: Mittelwerte und Mediane für die Überlebenszeit

\begin{tabular}{|c|c|c|c|}
\hline \multicolumn{2}{|l|}{ Mittelwert } & \multicolumn{2}{|c|}{$95 \%$ - } \\
& & \multicolumn{2}{c|}{ Konfidenzintervall } \\
\cline { 3 - 4 } & \multirow{2}{*}{ Standard- } & Untere & Obere \\
fehler & Grenze & Grenze \\
\hline 24,696 & 1,335 & 22,079 & 27,314 \\
\hline
\end{tabular}

Die geschätzte Überlebenszeit beträgt 24,7 Jahre.

\subsubsection{Patienten mit bzw. ohne Benzodiazepine}

In der Kontrollgruppe verstarben 33 Patienten, somit überlebten 84,1\%. In der Gruppe der benzodiazepinabhängigen Patienten verstarben 58 Patienten, 25 Patienten mehr als in der Kontrollgruppe und somit überlebten nur 72,0 \% (Tabelle 4.11, Diagramm 4.6).

Tabelle 4.11: Mortalität mit bzw. ohne Benzodiazepine

\begin{tabular}{|l|c|c|c|c|}
\hline \multirow{2}{*}{ Gruppe } & Gesamtzahl & $\begin{array}{c}\text { Anzahl der } \\
\text { Ereignisse }\end{array}$ & \multicolumn{2}{|c|}{ Zensiert } \\
\hline & $\mathrm{N}$ & Prozent & $\mathrm{N}$ & Prozent \\
\hline Kontrolle & 207 & 33 & 175 & $84,1 \%$ \\
\hline Benzodiazepine & 207 & 58 & 150 & $72,0 \%$ \\
\hline Gesamt & 414 & 89 & 325 & $78,5 \%$ \\
\hline
\end{tabular}


Diagramm 4.6: Mortalität mit bzw. ohne Benzodiazepine

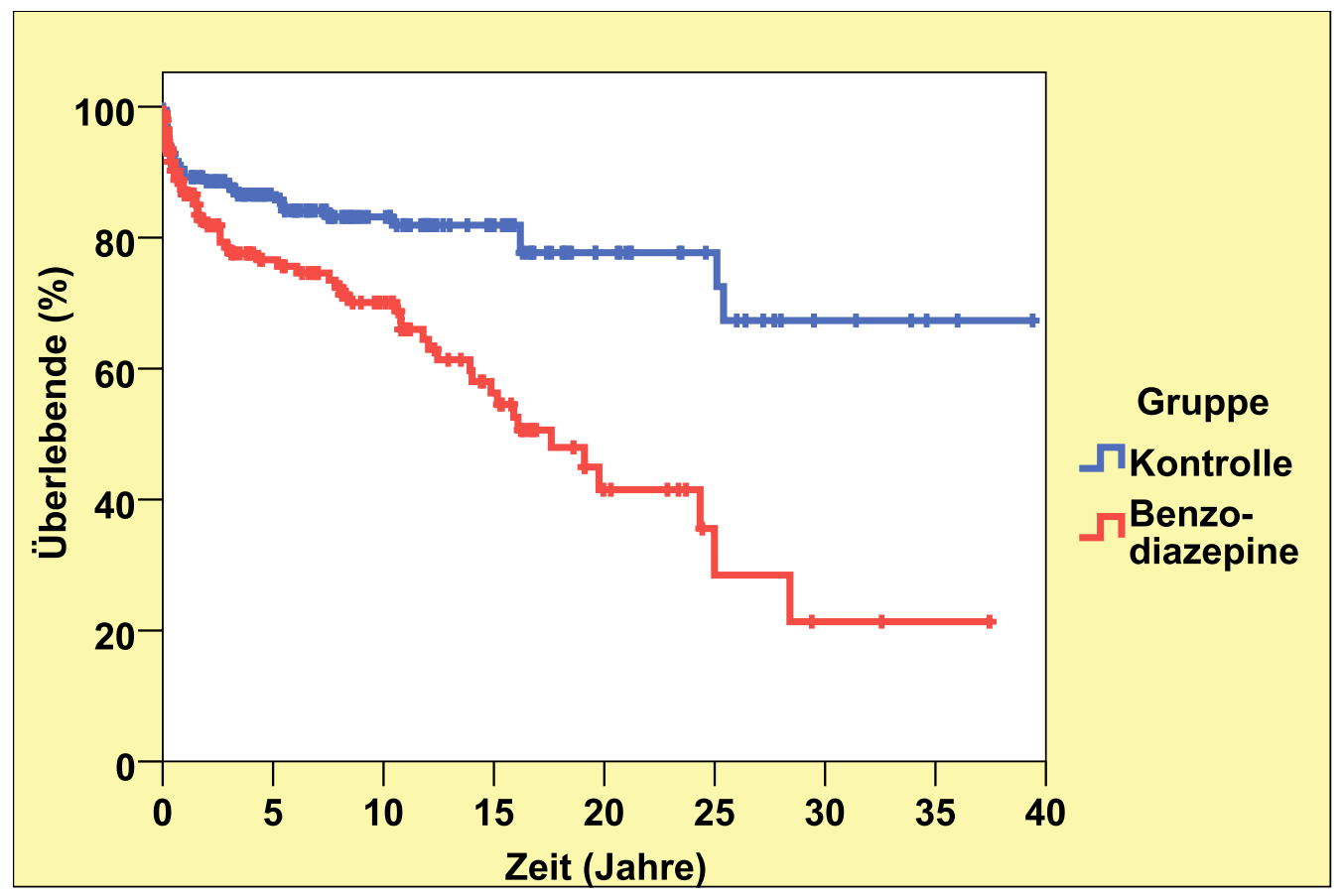

Die Gruppe mit Benzodiazepinabhängigkeit hatte eine eindeutig höhere Mortalität ( $27,5 \%$ vs. $15,5 \%$, wobei die geschätzte Überlebenszeit deutlich kürzer war als in der Kontrollgruppe (Tabelle 4.12) (18,3 Jahre vs. 30,3 Jahre).

Tabelle 4.12: Mittelwerte für die Überlebenszeit (Jahre)

\begin{tabular}{|c|c|c|c|c|}
\hline \multirow[t]{3}{*}{ Gruppe } & \multicolumn{4}{|l|}{ Mittelwert } \\
\hline & \multirow[b]{2}{*}{ Schätzer } & \multirow[b]{2}{*}{$\begin{array}{c}\text { Standard- } \\
\text { fehler }\end{array}$} & \multicolumn{2}{|c|}{$\begin{array}{c}\text { 95\%- } \\
\text { Konfidenzintervall }\end{array}$} \\
\hline & & & $\begin{array}{l}\text { Untere } \\
\text { Grenze }\end{array}$ & $\begin{array}{l}\text { Obere } \\
\text { Grenze }\end{array}$ \\
\hline Kontrolle & 30,277 & 1,560 & 27,219 & 33,335 \\
\hline Benzodiazepine & 18,335 & 1,677 & 15,048 & 21,621 \\
\hline Gesamt & 24,696 & 1,335 & 22,079 & 27,314 \\
\hline
\end{tabular}




\subsubsection{Mortalität in Abhängigkeit vom Geschlecht}

Von insgesamt 272 weiblichen Patienten verstarben 54. Somit überlebten 80,2\%. Von 142 männlichen Patienten verstarben 35. Mit 75,4\% überlebten etwas weniger Männer als Frauen (Diagramm 4.7, Tabellen 4.13 und 4.18).

Diagramm 4.7: Mortalität bei Männern und Frauen

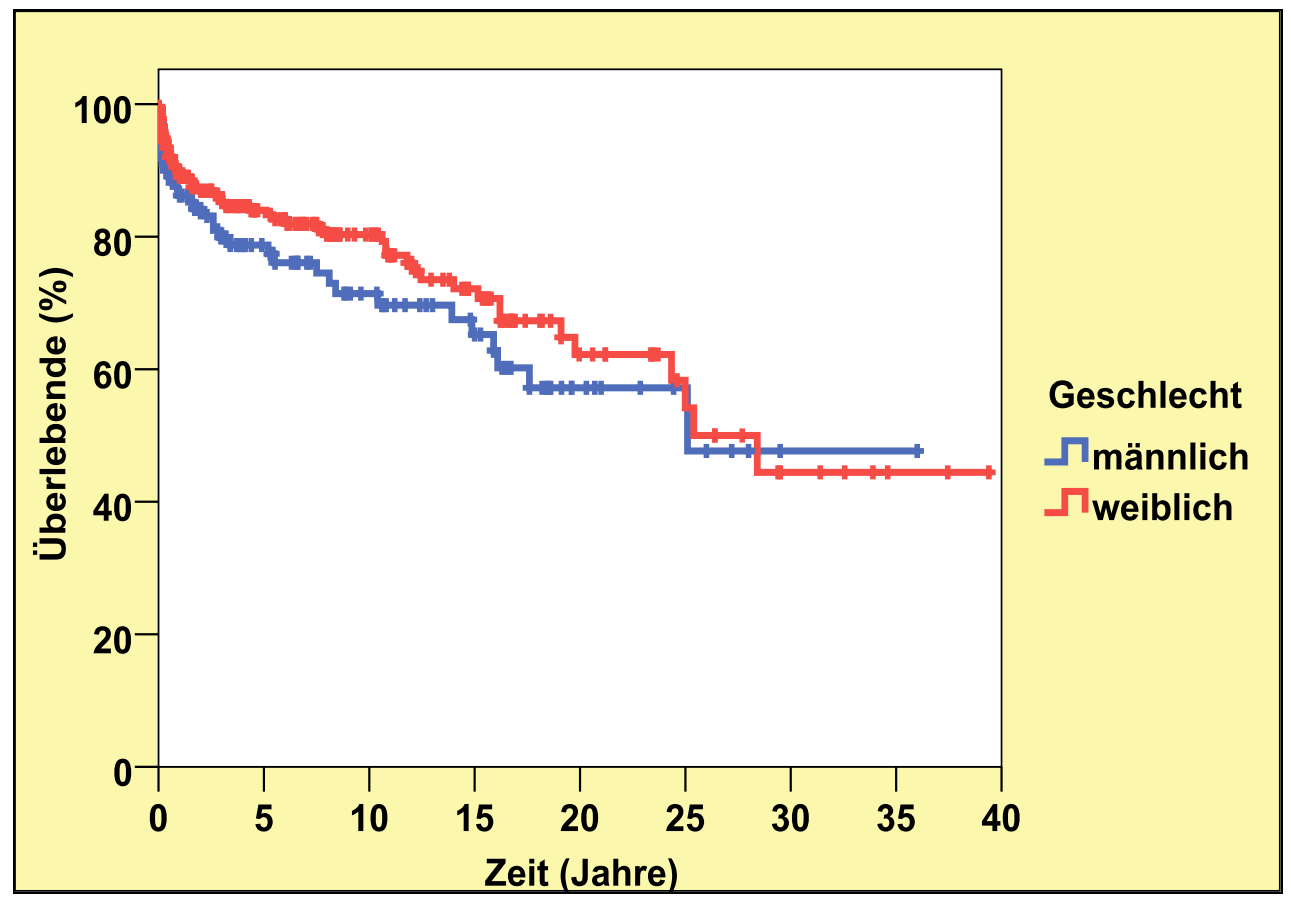

Tabelle 4.13: Sterblichkeit im Geschlechtervergleich

\begin{tabular}{|l|c|c|c|c|c|c|}
\hline Gruppe & $\mathrm{N}$ & weiblich & männlich & Quotient m/w & Gesamt & $\begin{array}{c}\text { Prozent } \\
\text { gesamt }\end{array}$ \\
\hline Benzo. & 207 & 35 & 22 & 1,59 & $57^{*}$ & 27,5 \\
\hline Kontrolle & 207 & 19 & 13 & 1,46 & $32^{\star *}$ & 15,5 \\
\hline Gesamt & 414 & 54 & 35 & 1,54 & 89 & 21,5 \\
\hline
\end{tabular}

*nach Aktenlage und Nachuntersuchung, ${ }^{* *}$ nach Aktenlage 
Aus der Tabelle 4.13 geht hervor, dass die Geschlechtsverteilung zwischen der Gesamtgruppe $(n=414)$ und der Kontrollgruppe $(n=207)$ sowie der Benzodiazepingruppe $(n=207)$ annähernd gleich ist. Der Mittelwert des Quotienten $\mathrm{m} / \mathrm{w}$ beträgt 1,53 . Hiervon weichen die Quotienten der drei Gruppen nicht wesentlich ab.

Tabelle 4.18: Mittelwerte für die Überlebenszeit (Jahre)

\begin{tabular}{|l|c|c|c|c|}
\hline Geschlecht & \multicolumn{2}{|l|}{ Mittelwert } & \multicolumn{2}{|c|}{$95 \%$ - } \\
& & & \multicolumn{2}{|c|}{ Konfidenzintervall } \\
\cline { 4 - 5 } & & Standard- \\
fehler & $\begin{array}{c}\text { Untere } \\
\text { Grenze }\end{array}$ & $\begin{array}{c}\text { Obere } \\
\text { Grenze }\end{array}$ \\
\hline Männlich & 22,442 & 1,889 & 18,740 & 26,144 \\
\hline Weiblich & 25,273 & 1,638 & 22,062 & 28,483 \\
\hline Gesamt & 24,696 & 1,335 & 22,079 & 27,314 \\
\hline
\end{tabular}

\subsection{Anmerkungen zur Anwendung der statistischen Methoden}

Die statischen Auswertungen wurden mit Hilfe von SPSS für Windows, Version 15.0 (SPSS Inc., U.S.A.) durchgeführt. Als Lageparameter der kontinuierlichen Variablen wurden Mittelwerte, als Streuungsparameter die Standardabweichungen gewählt.

Die kontinuierlichen Variablen wurden mittels des Kolmogorov-Smirnov-Tests hinsichtlich ihrer Verteilung überprüft. Während einige der getesteten Variablen nicht normalverteilt waren (Kolmogorov-Smirnov-Test: $p<0,05$ ), widersprachen andere der Annahme einer Normalverteilung nicht (Kolmogorov-Smirnov-Test: $p \geq 0,05$ ). Bei den Mittelwertvergleichen wurden daher Tests für normalverteilte Stichproben und nichtparametrische Tests für nicht normalverteilte Stichproben herangezogen. Beim Vergleich von zwei unabhängigen, normalverteilten Stichproben wurde der t-Test verwendet, während bei nicht normalverteilten 
Stichproben der Mann-Whitney-U-Test als nichtparametrisches Verfahren durchgeführt wurde.

Die kategorisierten Daten dagegen wurden mit Hilfe des Chi-Quadrat-Tests bzw. des exakten Tests nach Fisher ausgewertet. Bei Verwendung des Chi-QuadratTests wurden die erforderlichen Testvoraussetzungen erfüllt, da bei allen Tests weniger als $20 \%$ der erwarteten Häufigkeit kleiner 5 war.

Bei den durchgeführten Inferenz-Tests wurde die Signifikanz zweiseitig überprüft, wobei für alle statistischen Tests ein $p$-Wert $<0,05$ als statistisch signifikant angenommen wurde.

Die Überlebensstatistiken wurden mit Kaplan-Meier-Analysen durchgeführt. Als Testverfahren beim Vergleich der Sterberaten wurde der Log-Rank-Test verwendet.

In den grafischen Darstellungen, die ebenfalls mit SPSS erstellt wurden, wurden zur Veranschaulichung der Mittelwerte Fehlerbalken verwendet, wobei als Streuungsmaß aufgrund der großen Streuungsbreite die Standardfehler aufgeführt wurden. Die kategorialen Daten wurden grafisch mit Hilfe von einfachen und gruppierten Balkendiagrammen dargestellt.

\subsection{Kasuistik}

Die damals 24-jährige Studentin stellte sich erstmalig Ende der 70er-Jahre im Notdienst der Psychiatrischen Universitätsklinik Göttingen vor. Sie litt an einer akuten Panikattacke, die sich während eines Spaziergangs entwickelt hatte. An Symptomen bestanden Unruhe, Herzschmerzen, Dyspnoe, Angst vor der Angst und Angst vor dem Alleinsein. Die Patientin erlebte ihre normale Pulsfrequenz und ihren normalen Blutdruck als beschleunigt bzw. erhöht. Häufig fühlte sie sich 
selbst den Puls. Drei Tage vor der Notfallvorstellung war sie aus der Frauenklinik entlassen worden (insgesamt 3 Aufenthalte wegen Adnexitis).

Familienanamnese und frühkindliche/kindliche Entwicklung waren eigenanamnestisch unauffällig.

Die Symptome einer Angststörung bestanden seit der Schulzeit, insbesondere nach einer handgreiflichen Auseinandersetzung mit einem befreundeten Klassenkameraden, der die Patientin würgte, was sie als tödliche Bedrohung erlebte. Die Symptome hatten sich nach dem Tod der Großmutter vor einigen Jahren weiter verstärkt. Neben den Panikattacken bestanden ausgeprägte Prüfungsängste, Krankheitsphobien und eine Zahnarztphobie.

Frühere Diagnosen waren Herzneurose, hyperkinetisches Herzsyndrom, Hypotonie und Neigung zur Sinustachykardie. Daneben waren rezidivierende Adnexitiden und eine multiple Antibiotikaallergie bekannt. Zur Behandlung waren früher eine Gruppentherapie und ein Betablocker eingesetzt worden.

Bei der ersten Notfallaufnahme wurde die vorläufige Diagnose einer herzphobischen Entwicklung gestellt und eine Überweisung in die psychotherapeutische Abteilung der Klinik eingeleitet. Die Zeit bis zum ersten Termin wurde mit Lorazepam (1 mg Tavor pro Tag) überbrückt. Die Patientin selbst wäre lieber in die Innere Medizin gegangen, die Psychiatrie einschließlich psychotherapeutischer Abteilung war ihr eigentlich nicht recht. Sie verweigerte auch eine stationäre Behandlung in der psychotherapeutischen Abteilung, wurde dort aber ambulant unter Fortsetzung der Lorazepambehandlung weiterbehandelt. Die Patientin gab an, nicht allein sein zu können. 
In der folgenden Zeit kam es zu insgesamt zehn freiwilligen, vollstationären Aufnahmen (z.T. notfallmäßig) in die Psychiatrie, immer häufiger mit Abhängigkeitsdiagnosen:

\begin{tabular}{|c|c|c|c|c|}
\hline Jahr & $\begin{array}{l}\text { Dauer } \\
\text { (Tage) }\end{array}$ & Entlassungsdiagnose & $\begin{array}{l}\text { Benzodiazepine } \\
\text { (vor Aufnahme) }\end{array}$ & $\begin{array}{l}\text { Benzodiazepine } \\
\text { (während) }\end{array}$ \\
\hline 1979 & 2 & Angstneurose & $1 \mathrm{mg}$ Lorazepam & Keine \\
\hline 1979 & 1 & Angstneurose & $2 \mathrm{mg}$ Lorazepam & Keine \\
\hline 1979 & 173 & Neurotische Entwicklung & Lorazepam & Keine \\
\hline 1979/1980 & 12 & $\begin{array}{l}\text { Angstneurose, } \\
\text { Steißbeinfraktur }\end{array}$ & Unbekannt & Keine \\
\hline 1981 & 1 & $\begin{array}{l}\text { Angstneurose, } \\
\text { Tavor-Abusus }\end{array}$ & Beruhigungsmittel & keine \\
\hline 1986 & 7 & Neurotische Entwicklung & Lorazepam & Keine \\
\hline 1986 & 3 & Neurotische Entwicklung & Lorazepam & Keine \\
\hline 1986 & 14 & $\begin{array}{c}\text { Angstneurose, } \\
\text { Benzodiazepin-Abusus }\end{array}$ & $4 \mathrm{mg}$ Lorazepam & Reduktion \\
\hline 1986/1987 & 89 & $\begin{array}{c}\text { Angstneurose, } \\
\text { Benzodiazepinabhängigkeit }\end{array}$ & $3 \mathrm{mg}$ Lorazepam & Keine Reduktion \\
\hline 1987 & 4 & $\begin{array}{c}\text { Angstneurose, } \\
\text { Benzodiazepinabhängigkeit }\end{array}$ & $\begin{array}{c}>2 \mathrm{mg} \\
\text { Lorazepam }\end{array}$ & 2 mg Lorazepam \\
\hline
\end{tabular}

Aufnahmegrund war mehrfach die Überweisung aus einer somatischen Klinik zum Benzodiazepinentzug. Bei diesen Aufenthalten wurde u.a. festgestellt, dass die Patientin wahllos Ärzte aufsucht, die voneinander nichts wussten. Die stationären Behandlungen waren erfolglos, auch der lange Aufenthalt im Jahr 1979 (173 Tage). Während dieser stationären Behandlung auf einer offenen Station wurde eine intensive Verhaltenstherapie durchgeführt, dazu wurden Betablocker und Multivitamintabletten verabreicht (die Patientin kam kachektisch). Der Terminus „Odyssee durch die Göttinger Kliniken“ wurde benutzt. Sie strebte eine Psychoanalyse an, die aber zum damaligen Zeitpunkt als kontraindiziert angesehen wurde. Sie wurde daher von mindestens drei Psychoanalytikern als Patientin abgelehnt. Unspezifische psychotherapeutische Maßnahmen (Gruppentherapie, entlastende Gespräche, respiratorisches Biofeedback) waren wirkungslos, ebenso Betablocker und Spasmolytika. 
Bei einer ambulanten Konsiliaruntersuchung durch einen Psychiater wurden 1992 folgende Diagnosen gestellt:

- Angstneurose mit Agoraphobie

- Benzodiazepinabhängigkeit (kein Abusus)

- Osteoporose durch krankheitsbedingte Inaktivität

- Alte LWK-Fraktur mit chronischen Rückenschmerzen.

Die Lorazepamdosis betrug zu diesem Zeitpunkt 6-10 mg pro Tag. Die Patientin akzeptierte den empfohlenen stationären Entzug mit desensibilisierender Verhaltenstherapie nicht.

Heute besteht eine ausgeprägte Benzodiazepinabhängigkeit vom Hochdosistyp (6-15 mg Lorazepam pro Tag), die bei Medikamentenreduktion oder gar Absetzen zu schweren Entzugserscheinungen führt. Trotz Medikation besteht eine so schwerwiegende Agoraphobie, dass die Patientin ihr Zimmer nicht verlassen kann, außer zu Notfallvorstellungen in somatischen Kliniken (Cholelithiasis, Harnwegsinfekte).

\section{Epikrise:}

Die 24-jährige Patientin stellte sich erstmals Ende der 70er-Jahre notfallmäßig in der Psychiatrischen Universitätsklinik Göttingen wegen einer typischen, akuten Panikattacke vor. Außerdem äußerte sie Angst vor der Angst und vor dem Alleinsein. Bei der Erstaufnahme wurde die „vorläufige Diagnose“ einer herzphobischen Entwicklung gestellt und bis zur Aufnahme in die Psychotherapeutische Abteilung mit $1 \mathrm{mg}$ Tavor/tgl. zur Überbrückung behandelt. Die Aufnahme in die Psychiatrische Abteilung lehnte die Patientin ab, deshalb wurde sie ambulant mit Lorazepam weiterbehandelt. Erste Untersuchungen von Puls und Blutdruck ergaben Normalbefunde. Die Patientin wurde mehrfach organisch vollständig durchuntersucht („Odyssee durch die Göttinger Kliniken“). Entsprechende Befunde (auch negative) liegen nicht vor, lediglich kurze Diagnosemitteilungen und Klinik-Entlassungsbriefe. Die Tatsache der fehlenden Krankheitseinsicht ist aus der Literatur bekannt (Poser W und Wegerer 2001, 
S. 205): „Dagegen fallen die isolierten Benzodiazepinsuchten [...] im Praxisalltag wegen ihres meist blanden Verlaufes kaum auf, ebenso wenig im klinischen Bereich“. Diverse Reaktionen von Patienten in der vorliegenden Studie, die eine Benzodiazepinabhängigkeit verneinten, obwohl sie deswegen stationär behandelt wurden, bestätigen dies (siehe Anhang 4). In der Folgezeit ließ sich die Patientin jedoch 10-mal freiwillig in die Psychiatrie stationär aufnehmen. Es wurden immer häufiger Abhängigkeitsdiagnosen gestellt, während die Grunddiagnose "Angstneurose" stets gleich blieb und immer wieder bestätigt wurde. Anfangs (1979) bekam die Patientin während 7 stationärer Aufenthalte bis 1986 keine Benzodiazepine verordnet, weil bereits 1981 ein schädlicher Lorazepamgebrauch (damals Abusus genannt) festgestellt worden war, der offensichtlich noch nicht in eine Abhängigkeit übergegangen war. Noch im Laufe des Jahres 1986 hatte sich aber der schädliche Benzodiazepingebrauch offensichtlich derart verstärkt, dass während des stationären Aufenthalts ein Entzug durch Reduktion der Dosis versucht wurde. Die vielmehr schwere Angstneurose wurde zunehmend für unbeeinflussbar gehalten. Seit 1986 nimmt die Patientin regelmäßig Benzodiazepine in steigender Dosierung (zuletzt 6-15 mg Lorazepam pro Tag), sodass nunmehr eine ausgeprägte Benzodiazepinabhängigkeit vom Hochdosistyp vorliegt, die bereits bei Reduktion der Dosis zu Entzugserscheinungen führt. Die Abhängigkeit wurde 1992 durch einen Psychiater erneut bestätigt. Dieser Fall zeigt die eher seltene Entwicklung zu einer Hochdosisabhängigkeit (Tagesdosis über 7,5 mg Lorazepam). In der Mehrzahl der Fälle bleibt es bei einer Normaldosisabhängigkeit (Ziegler 1991).

\section{Diskussion}

Die Nachuntersuchung zum Verlauf per Fragebogen war eher ein Misserfolg (obwohl durch die wenigen antwortenden Patienten oder Angehörigen oft wertvolle Hinweise gegeben wurden): Von den angeschriebenen 184 Patienten haben 81 die Einverständniserklärung zurückgesandt ( $=44 \%$ Rücklaufquote). Davon haben 34 das Einverständnis verweigert (= $42 \%$ ). Somit bleiben 47 Patienten, die den 
Fragebogen erhalten hatten. Davon kamen 43 zurück (= $91 \%$ ). Insgesamt bilanziert haben also von 184 letztlich nur 43 den Fragebogen ausgefüllt (= $23 \%$ ). Folglich ist die „Rücklaufquote“ vor allem eine Frage der Definition, da mit dem Versenden der Anfragebriefe mehr beantwortete Fragebogen erwartet bzw. erwünscht wurden. $\mathrm{Zu}$ beachten ist auch die hohe Mortalität der Benzodiazepinabhängigen. Verstorbene Patienten können natürlich keinen Fragenbogen ausfüllen. Deutlich erfolgreicher waren die Auskünfte der Einwohnermeldeämter. Durch sie konnte bei fast allen Patienten festgestellt werden, ob sie noch lebten oder bereits verstorben waren. Außerdem konnte das für die Kaplan-Meier-Analyse wichtige Todesdatum eruiert werden. Auch die Durchsicht der Krankengeschichte des Hauses war informativ. Fast alle Patienten (Benzodiazepinabhängige und Kontrollen) waren mehrfach aufgenommen worden. Damit konnte der Verlauf in den meisten Fällen aus der Krankengeschichte entnommen werden.

Wesentliches Ergebnis dieser Arbeit ist die Feststellung, dass Benzodiazepinabhängige mit komorbider Erkrankung eine deutlich höhere Mortalität aufweisen als ähnlich Erkrankte ohne Benzodiazepinabhängigkeit. Dies konnte vor allem durch die Auswertung der Krankengeschichten im Langzeitverlauf nachgewiesen werden. Viele Patienten kamen mehrfach in ambulante oder stationäre Behandlung der Klinik. Dies betraf vor allem solche Patienten, die nicht abstinent wurden, aber überlebten. Dieses Ergebnis scheint dem einer früheren Untersuchung der Arbeitsgruppe um Poser W zu widersprechen. Damals wurde keine erhöhte Mortalität Benzodiazepinabhängiger im Vergleich zu parallelisierten Kontrollen gefunden (Strehlow et al. 1988). Jedoch wird bei der Inspektion der Survivalkurven der vorliegenden Untersuchung (Diagramm 4.6) deutlich, dass die Übersterblichkeit der Benzodiazepinabhängigen in den ersten beiden Beobachtungsjahren noch nicht besteht, sondern erst im weiteren Verlauf deutlich wird. Damit konnte die frühere Untersuchung den Unterschied der beiden Kollektive noch nicht finden, da die Nachbeobachtungszeit wesentlich kürzer war. Außerdem waren die Patientenkollektive der früheren Untersuchung wesentlich kleiner (Strehlow et al. 1988). 
Auch wenn nachgewiesen werden konnte, dass in der Benzodiazepingruppe die Mortalität signifikant höher war als in der Kontrollgruppe, lässt dies keinen sicheren Schluss auf einen kausalen Zusammenhang zu, sondern ist zunächst nur als Tatsache zu verzeichnen. Nicht jeder Todesfall eines Suchtkranken ist unmittelbar auf den Substanzkonsum oder den gleichzeitigen Konsum mehrerer Substanzen oder ein Entzugssyndrom zurückzuführen (Gibson et al. 2011, Jenkins 2000, Kuo et al. 2011, Nyhlén et al. 2011). Andere Zusammenhänge zwischen Benzodiazepinkonsum und -abhängigkeit sind denkbar und wahrscheinlich wichtiger:

1. So könnte der Dauerkonsum von Benzodiazepinen die Suizidalität und die Suizidrate erhöhen. Das stellten Neutel und Patten (1997) für Benzodiazepinkonsumenten fest. In unserer Untersuchung mit Benzodiazepinabhängigen (Neigung zur Dauereinnahme) dürfte die Suizidalitätserhöhung sogar verstärkt aufgetreten sein. In einer früheren Publikation der Arbeitsgruppe wurden relativ häufig Suizide als Todesursache bei Arzneimittelabhängigen festgestellt (Poser W et al. 1997). Allerdings waren in der vorliegenden Untersuchung die Todesursachen nur selten bekannt. Trotzdem gehen wir davon aus, dass eine Erhöhung der Suizidalität ein wichtiger Teilfaktor für die erhöhte Gesamtmortalität ist.

2. Benzodiazepine führen allein bei Gesunden praktisch nie zum Tod durch Intoxikationen. Jedoch können bestimmte Vorkrankheiten wie „COPD“ oder „Myasthenia gravis“ die Toxizität erhöhen. Diese Vorkrankheiten sind an sich Kontraindikationen für die Gabe von Benzodiazepinen (s. Fachinformationen); die Kontraindikationen werden aber oft nicht eingehalten. In unserer Untersuchung haben wir keine Patienten mit „Myasthenia gravis“ gefunden, jedoch einige starke Raucher. Deshalb könnte eine erhöhte Benzodiazepintoxizität bei „COPD-Patienten“ eine weitere Mitursache für die gefundene erhöhte Mortalität bei Benzodiazepinabhängigen sein. Leider enthielten die Krankengeschichten nur selten Hinweise auf den Zigarettenkonsum, sodass wir nicht 
abschließend feststellen konnten, ob die Benzodiazepinabhängigen stärker rauchten als die Kontrollen.

3. Weiterhin können Benzodiazepine das Risiko von Stürzen und Unfällen bei Hochbetagten erhöhen (Koski et al. 2002, Titler et al. 2011). Selbst in der Untersuchung von Vinkers et al. (2003) mit Benzodiazepinkonsumenten im Alter von 85 Jahren und darüber, in der die Gesamtmortalität der Benzodiazepinkonsumenten nicht signifikant erhöht war, fanden sich unter diesen mehr Frakturen. Dies dürfte in dieser Untersuchung mit Abhängigen, die zu höheren Benzodiazepindosen neigen, sehr wohl eine Rolle gespielt haben. Auch diese Frage ist aber ohne Kenntnis der Todesursachen nicht zu entscheiden.

4. Mehrfach wurde gezeigt, dass Benzodiazepinkonsumenten ein erhöhtes Risiko für Straßenverkehrsunfälle haben (Karjalainen et al. 2010, Orriols et al. 2009). Das gilt ganz besonders für solche, die gleichzeitig Alkohol trinken oder andere sedierende Suchtstoffe nehmen (Karjalainen et al. 2010). Das erhöhte Risiko besteht nicht nur für Straßenverkehrsunfälle, sondern auch für andere Unfälle (French et al. 2005). Es erscheint denkbar, dass dieser Faktor bei Benzodiazepinabhängigen mit ihrer Neigung zu höheren Dosen sogar noch wichtiger ist. Ohne Kenntnis der Todesursachen ist auch diese Frage aber nicht zu entscheiden.

5. Benzodiazepine können das Risiko für vital bedrohliche Intoxikationen bei gleichzeitigem Konsum mit anderen Suchtstoffen erhöhen, z.B. Alkohol oder Opioide (Green et al. 2011, Koski et al. 2002). Solche Patienten wurden aber nicht in unsere Untersuchung einbezogen, deshalb dürfte diese Denkmöglichkeit hier keine Rolle spielen. Benzodiazepinabhängige mit zusätzlichem Alkohol- und Opioidkonsum sind in einer Untersuchung mit isoliert Benzodiazepinabhängigen bzw. parallelisierten Kontrollen natürlich auszuschließen. 
6. Die Mortalität von niereninsuffizienten Patienten mit Benzodiazepinkonsum (unter diesen waren auch Patienten, die an "COPD“ erkrankt waren) ist im Vergleich zu Nichtkonsumenten erhöht (Winkelmayer et al. 2007). Dies lässt sich vor allem auf die Benzodiazepineinnahme von "COPD-Patienten“ zurückführen, die auch mehr rauchen als Nichteinnehmer, was sich mit der bekannten atemdepressiven Wirkung von Benzodiazepinen bei „COPDPatienten" erklären lässt. Daher muss damit gerechnet werden, dass in jedem Kollektiv mit „COPD-Patienten“ Benzodiazepine die Mortalität erhöhen. In den vorliegenden Kollektiven könnten die Benzodiazepinabhängigen mehr und häufiger geraucht haben als die Kontrollen. Daher wird davon ausgegangen, dass dieser Faktor zu der erhöhten Mortalität der Benzodiazepinabhängigen beigetragen haben könnte. Diese Frage kann aber nicht geklärt werden, weil der Tabakkonsum bei stationären Patienten der Psychiatrie nur sehr unvollständig registriert wird.

7. In der Benzodiazepingruppe könnten lebensgefährliche oder tödliche Entzugssyndrome aufgetreten sein, die in der Kontrollgruppe naturgemäß nicht auftreten. Diesen Faktor halten wir aber für irrelevant, da Todesfälle an Entzugssyndromen heute eine Rarität darstellen. Ein solcher Fall ist in unserer Klinik bei Benzodiazepinabhängigen nicht beobachtet worden, im Gegensatz zu gelegentlichen Todesfällen im Alkoholentzug.

Eine statistische Auswertung der Fragebogen ist nicht sinnvoll. Einerseits ist ihre Anzahl im Verhältnis zum untersuchten Patientenkollektiv zu gering $(20,8 \%)$. Möglicherweise hatten viele $(76,6 \%)$ der Angeschriebenen Angst vor weiterer „Belästigung“. Auch fehlendes Interesse, da es ihnen gut ging, wäre denkbar. Uns erscheinen auch die angegebenen Zahlen zumindest für Nikotin- und Alkoholkonsum bzw. pathologischen Konsum nicht durchschaubar. Besonders den Anmerkungen in den Fragebogen ist zu entnehmen, dass ein Teil der Patienten eine starke Abneigung gegenüber der Beantwortung der Fragen erkennen ließ. Ob nun aus Unzufriedenheit oder aufgrund von schlechten Erfahrungen, sei dahingestellt. Dennoch sind sie sehr interessant. Dies gilt insbesondere für die schriftlichen Kommentare (siehe Anhang 4). 
Die Ergebnisse dieser Arbeit (einschließlich der Fragebogenauswertungen) können als Ausgangspunkt eines (aufwendigen) Forschungsprojektes zu den Ursachen der erhöhten Mortalität von Benzodiazepinabhängigen dienen. Ein solches Projekt müsste einen langen Zeitraum nach der Erstdiagnose umfassen und die Todesursachen einschließen. Außerdem müsste es das Augenmerk auf die weitere Benzodiazepinexposition nach Erstdiagnose erfassen und in die Auswertung einbeziehen. Wenn die Vermutung stimmt, dass vor allem Suizide und Unfälle der Grund für die erhöhte Mortalität Benzodiazepinabhängiger sind, würde eine Analyse der späteren Benzodiazepinexposition den kausalen Zusammenhang zwischen Fortbestehen der Abhängigkeit und der Mortalität belegen.

\section{Zusammenfassung}

In der vorliegenden Studie wurde erstmalig die isolierte Benzodiazepinabhängigkeit im Langzeitverlauf im Vergleich zu Kontrollen mit einem vergleichbaren Krankheitsspektrum über einen langen Zeitraum und an einem großen Kollektiv untersucht. Es wurden 207 isoliert benzodiazepinabhängige Patienten mit insgesamt 1.127,6 Patientenjahren und ebenfalls 207 Kontrollen untersucht. Das Gesamtkollektiv wurde insgesamt über einen Zeitraum von 46 Jahren (erste Untersuchung in der Klinik bis letzter Fragebogeneingang) beobachtet.

Die längste Zeit (time under observation) eines Patienten betrug 39,4 Jahre. Im Durchschnitt wurden die Patienten sieben Jahre lang beobachtet. Die benzodiazepinabhängigen Patienten waren im Schnitt 2,4 Jahre weniger unter Beobachtung als ihre Kontrollen mit der gleichen Grunderkrankung. Das hängt sicherlich mit der höheren Sterblichkeit dieser Gruppe zusammen.

Bei Survivalanalysen werden Verstorbene am Todestag aus der Untersuchung genommen. 
Gezeigt wurde erneut, dass es sich bei der Benzodiazepinabhängigkeit um eine Abhängigkeit des mittleren Lebensalters handelt. Das Durchschnittsalter bei Erstdiagnose in dieser Untersuchung betrug 48,2 Jahre. Für den Erkrankungsbeginn (der Abhängigkeit, nicht der Grunderkrankung) wurde ein Durchschnittsalter von 42 Jahren gefunden (Poser W 1991). Was in dieser Studie erneut bestätigt wird, ist die Tatsache, dass Frauen häufiger an einer isolierten Benzodiazepinabhängigkeit erkranken als Männer (annähernd doppelt so häufig).

Das Hauptanliegen dieser Studie war zu klären, ob eine isolierte Benzodiazepinabhängigkeit zu einer erhöhten Mortalität führt. Nach Auswertung der Daten ergab sich, dass die Gruppe der isoliert Benzodiazepinabhängigen im Vergleich zur Kontrollgruppe eine hochsignifikant höhere Mortalität aufwies. Dies wurde so in bisherigen Untersuchungen noch nicht beschrieben. Denkbare Ursachen für die erhöhte Mortalität werden diskutiert. Daraus ergeben sich Hinweise für eine (aufwendigere) Studie zu den Gründen der erhöhten Mortalität. 


\section{Anhänge}

\subsection{Anhang 1 (Grunderkrankungen)}

\section{Häufigkeiten der Grunderkrankungen mit Geschlechterverteilung}

(Ausgangspunkt der Benzodiazepinverordnungen)

\begin{tabular}{|c|c|c|}
\hline Diagnose & $\mathbf{W}$ & $\mathbf{M}$ \\
\hline Neurasthenisches Syndrom & & 1 \\
\hline Panikattacken & 4 & 2 \\
\hline Schizoaffektive Psychose & & 1 \\
\hline Nervöse Magenbeschwerden & & 1 \\
\hline Bipolar-affektive Störung/Zyklothymie & $1 / 1$ & $1 / 3$ \\
\hline Soziale Steuerungsschwäche bei Chorea Huntington & & 1 \\
\hline Psychischer Erschöpfungszustand & 2 & \\
\hline „Ehekonflikt” & 2 & \\
\hline Parasuizidale Kurzschlussreaktion & & 1 \\
\hline $\begin{array}{l}\text { „automatisch Benzodiazepin im KH verabreicht } \\
\text { bekommen, obwohl Schlaf in Ordnung war” }\end{array}$ & 1 & 1 \\
\hline Ängstlich-depressive Symptomatik & 1 & \\
\hline $\begin{array}{l}\text { Psychische Dekompensation i. S. einer ängstlich- } \\
\text { agitierten Depression bei Prostata-Karzinom }\end{array}$ & & 1 \\
\hline Phobisch-anankastische Depression & 1 & \\
\hline Agitierte Depression bei prämorbid unreifer Persönlichkeit & 1 & \\
\hline „zur Hypertoniebehandlung” & 1 & \\
\hline Posttraumatische Belastungsstörung & 1 & \\
\hline Innere Unruhe & 1 & 4 \\
\hline Psychotische Dekompensation & 1 & \\
\hline
\end{tabular}




\begin{tabular}{|c|c|c|}
\hline Schlafstörungen & 23 & 12 \\
\hline Erregungszustände & 4 & 1 \\
\hline $\begin{array}{l}\text { Funktionelle Oberbauchbeschwerden bei rez. Ulcus } \\
\text { ventriculi und duodeni }\end{array}$ & & 1 \\
\hline $\begin{array}{l}\text { Depressive Verstimmungszustände aufgrund } \\
\text { psychomotorischer Anfallsleiden }\end{array}$ & 2 & \\
\hline Multiple Phobien & 4 & 3 \\
\hline Neurotische Depression & 2 & 2 \\
\hline Neurotische Persönlichkeitsstruktur & 1 & \\
\hline $\begin{array}{l}\text { Subdepressives Zustandsbild bei primär } \\
\text { psychasthenischer Persönlichkeit }\end{array}$ & 1 & \\
\hline Borderline-Syndrom & 3 & \\
\hline Torticollis spasticus & 1 & 1 \\
\hline Zwangsneurose & 1 & 3 \\
\hline Händetremor & 1 & \\
\hline Bulimie, DD: Anorexie & 1 & \\
\hline Colitis ulcerosa & 1 & \\
\hline Angstkrankheit/-neurose & 28 & 18 \\
\hline $\begin{array}{l}\text { Psychosomatische Beschwerden (herzphobische } \\
\text { Beschwerden etc.) }\end{array}$ & 4 & 8 \\
\hline Zwanghaft-depressive Persönlichkeitsstruktur & & 1 \\
\hline Entlastungsdepression & 1 & \\
\hline Epilepsie & 1 & 1 \\
\hline Rez. endogene Depression & 24 & 13 \\
\hline Spätdepression bei Verschuldungswahn & 1 & \\
\hline Depressiv-schizoide Neurose/depressive Neurose & $1 / 1$ & \\
\hline Involutionsdepression & 5 & 1 \\
\hline Reaktive Depression & 10 & 3 \\
\hline Schizophrenie & 1 & 1 \\
\hline Chronische Rückenschmerzen & 3 & 3 \\
\hline Somatische Depression & 3 & 1 \\
\hline
\end{tabular}




\section{Nebendiagnosen}

Psychiatrische Erkrankungen

\begin{tabular}{|c|l|}
\hline $\begin{array}{c}\text { Anzahl der } \\
\text { Patienten }\end{array}$ & Erkrankungen \\
\hline 4 & Asthenische Persönlichkeit \\
\hline 18 & Phobisch-depressive Neurose \\
\hline 2 & Phobisch-hysterische Charakterneurose \\
\hline 6 & Ängstlich-agitierte Depression \\
\hline 8 & Phobische Angstzustände \\
\hline 11 & Angstneurose \\
\hline 1 & Kontrollzwänge \\
\hline 5 & Zwangsstörungen \\
\hline 14 & Z. n. Suizidversuch unbekannter Ursache \\
\hline 2 & Chronisch suizidal \\
\hline 4 & Maniforme Psychose \\
\hline 1 & Paranoid-halluzinatorische Psychose \\
\hline 12 & Depressives Syndrom \\
\hline 7 & Agoraphobie \\
\hline 1 & Sadomasochistisch-phobische Partnerbeziehung \\
\hline 4 & Dementielles Syndrom \\
\hline 4 & Hirnorganischer Abbauprozess unklarer Genese \\
\hline 16 & Zyklothymie/bipolare Störung \\
\hline 1 & Involutions-/endogene Depression \\
\hline 1 & Depression bei anankastischer Persönlichkeitsstruktur \\
\hline & Schlafstörungen (2) (1 Hypersomnie, 4 chronische \\
\hline 1 & Somatisierte Depression \\
\hline 1 & Anorexie \\
\hline 1 & \\
\hline 1 & \\
\hline 1 &
\end{tabular}




\begin{tabular}{|l|l|}
\hline 1 & V. a. Hebephrenie \\
\hline 2 & Adoleszenzkrise \\
\hline 2 & Somatisierungsstörungen \\
\hline 2 & V. a. Manie \\
\hline 2 & Schizophrenie \\
\hline 4 & Herzphobische Entwicklung bei Versagenszustand \\
\hline 1 & Nervöser Erschöpfungszustand \\
\hline 1 & Polytoxikomanie \\
\hline 1 & Antriebsdefizit \\
\hline 1 & Affektstörung \\
\hline 4 & Reaktive Depression \\
\hline 4 & HOPS (1x bei Raumforderung, Meningeom) \\
\hline 4 & Borderline-Störung \\
\hline 1 & Devitalisierungssyndrom \\
\hline 4 & Anpassungsstörung \\
\hline 1 & Paradoxe Reaktion auf Benzodiazepine \\
\hline
\end{tabular}

$\underline{\text { Internistische Erkrankungen }}$

\begin{tabular}{|c|l|}
\hline 16 & Diabetes mellitus \\
\hline 16 & $\begin{array}{l}\text { Euthyreote Struma/Z. n. Struma-OP/Hyper-/Hypo- } \\
\text { parathyreoidismus }\end{array}$ \\
\hline 28 & Arterielle Hypertonie \\
\hline 13 & Lungenerkrankungen (COPD/Asthma/Emphysem) \\
\hline 23 & $\begin{array}{l}\text { Funktionelle Herzkreislaufstörungen/KHK/Angina } \\
\text { pectoris/Herzrhythmusstörungen }\end{array}$ \\
\hline 12 & Hyperlipoproteinämie \\
\hline 22 & Nieren-/Milz-/Leber-/Pankreaserkrankungen \\
\hline 1 & Aortenaneurysma \\
\hline 17 & Gastritis/Magenulzera/Z. n. Magen-OP/Colon irritabile \\
\hline
\end{tabular}


Neurologische Erkrankungen

\begin{tabular}{|c|l|}
\hline 4 & M. Parkinson \\
\hline 7 & Multifaktorieller Kopfschmerz \\
\hline 1 & Vasomotorischer Kopfschmerz \\
\hline 1 & Migräne \\
\hline 3 & Restless legs \\
\hline 1 & Enzephalomyelitis disseminata \\
\hline 1 & Trigeminusneuralgie \\
\hline 1 & Epilepsie \\
\hline 3 & Z. n. Schlaganfall \\
\hline 1 & Globale Hirnatrophie \\
\hline 1 & M. Alzheimer \\
\hline 1 & Enzephalopathie (Dialyse, Benzodiazepinentzug) \\
\hline 1 & Vaskuläre Enzephalopathie \\
\hline 1 & Zerebrale Sklerose \\
\hline
\end{tabular}

Orthopädische Erkrankungen

\begin{tabular}{|c|l|}
\hline 4 & M. Bechterew \\
\hline 18 & BSV/degenerative Veränderungen der WS \\
\hline 2 & Osteoporose \\
\hline 1 & Myopathie \\
\hline 1 & Dupuytren-Kontraktur \\
\hline 8 & Arthrose \\
\hline 1 & Karpaltunnelsyndrom \\
\hline 2 & Myalgien \\
\hline
\end{tabular}


Dermatologische Erkrankungen

\begin{tabular}{|c|l|}
\hline 1 & Hyperhidrosis \\
\hline 1 & Alopezie \\
\hline 3 & Prurigo/Dermatitis \\
\hline 1 & Hyperkeratose \\
\hline 1 & Psoriasis vulgaris \\
\hline 1 & Neurodermitis \\
\hline
\end{tabular}

Urologische und gynäkologische Erkrankungen

\begin{tabular}{|c|l|}
\hline 7 & Prostataadenom/Orchiektomie \\
\hline 6 & Reizblase/hypotone Blase/Harninkontinenz \\
\hline 1 & Balanitis \\
\hline 1 & Adnexitis \\
\hline 2 & Vasektomie/Sterilisation \\
\hline 4 & Interruptio/Bauchhöhlenschwangerschaft/Ovarial-Ca \\
\hline 8 & Hysterektomie/Collum-Ca/Tuboovarialabszess \\
\hline 5 & Mamma-Ca \\
\hline
\end{tabular}

\section{Vorhergehende Suchten}

Von 69 der 207 benzodiazepinabhängigen Patienten waren vorangehende Suchtkrankheiten (schädlicher Gebrauch oder Abhängigkeit) bekannt. Zum Zeitpunkt der Erstuntersuchung war entsprechend den Ausschlusskriterien nur eine der beiden Benzodiazepinsuchten = „substance use disorders“ (schädlicher Gebrauch oder Abhängigkeit) bekannt (Nikotin nicht berücksichtigt). 


\begin{tabular}{|l|c|}
\hline „Suchtstoff” & Anzahl der Patienten \\
\hline Barbiturate & 7 \\
\hline Nikotin & 40 \\
\hline Alkohol & 12 \\
\hline Cannabis & 2 \\
\hline Anxiolytika & 1 \\
\hline Mischanalgetika & 1 \\
\hline Laxanzien & 1 \\
\hline Hypnotika & 1 \\
\hline Antidepressiva & 2 \\
\hline Opioide & 1 \\
\hline Haschisch & 1 \\
\hline
\end{tabular}

Kombinationen:

1x Nikotin + Alkohol

$1 x$ Nikotin + Cannabis

1x Barbiturate + Alkohol

1x Barbiturate + Anxiolytika

$1 \mathrm{x}$ Nikotin + Analgetika

1x Nikotin + Opioide

1x Haschisch + Alkohol + Cannabis + Nikotin 


\subsection{Anhang 2 (Fragebogen)}

Heutiges Datum:

(bitte eintragen)

Code-Nr.:

\section{Fragebogen zum Langzeitverlauf}

1. Waren Sie überhaupt jemals abhängig oder suchtkrank?

Ja $\square \quad$ Nein $\square$

2. In welchem Jahr hat Ihre Suchtkrankheit nach Ihrer Schätzung begonnen?

3. Welcher Suchtstoff war der erste in Ihrem Leben?

4. In welchem Jahr mussten Sie erstmals wegen Ihrer Sucht ins Krankenhaus?

5. Wie viele stationäre Entzüge haben Sie wegen einer Abhängigkeit gemacht?

6. Wann hatten Sie Ihren ersten stationären Entzug?

7. Wie viele Langzeittherapien haben Sie gemacht?

8. Welche Suchtstoffe haben Sie jemals genommen? 
9. Wie war Ihre Höchstdosis (im ganzen Leben) an „Benzodiazepinen“ = Schlafund Beruhigungsmittel?

10. Wurde Ihre Suchterkrankung durch eine andere Grundkrankheit ausgelöst (z.B. Migräne, Depression etc.)?

11. Wie viel rauchen Sie zurzeit täglich?

Seit wann rauchen Sie?

12. Welche Medikamente haben Sie gestern eingenommen?

13. War Alkohol jemals ein Problem für Sie?

Wenn ja, wie lange?

14. Trinken Sie zurzeit zu viel Alkohol? Ja $\square$ Nein $\square \quad$ Überhaupt keinen Wenn ja, wann zuletzt?

15. Gab/gibt es Suchtkrankheiten in Ihrer Herkunftsfamilie? $\quad$ Ja $\square \quad$ Nein $\square$ Wenn ja, welche und wer?

16. Welche Folgen Ihrer Suchtkrankheiten haben Sie (gehabt)?

17. Welche Entzugserscheinungen hatten Sie bisher (im ganzen Leben)? 
18. Wenn Sie jetzt nüchtern (suchtstofffrei) sind, was hat Ihnen am meisten geholfen?

19. Mein Suchtstoffproblem hat sich seit der Behandlung in der Klinik folgendermaßen entwickelt (bitte nur eine Antwort):

- Seither „trocken“ und bis heute kein Rückfall

- Sehr gebessert (kleine Rückfälle, aber nicht stationär behandelt)

- Gebessert (lang anhaltende Rückfälle mit stationärem Entzug, aber auch nüchterne Zeiten)

- Gebessert (ich trinke wie vorher/nehme kontrolliert Tabletten)

Wie lange schon?

- Unverändert (ich nehme wie vorher ein)

- Verschlimmert (ich brauche mehr als früher)

- Ich trinke nicht mehr, nehme aber Tabletten.

Welche Tabletten?

- Ich nehme keine Tabletten mehr, trinke aber.

20. Meine Besserung verdanke ich vorwiegend (mehrere Antworten möglich):

- Dem stationären Entzug

- Der stationären Therapie

- Der stationären Langzeittherapie

- Meinem Psychiater

- Der Suchtberatungsstelle

- Der Selbsthilfegruppe

- Meiner Familie/meinem Partner

- Freunden und Bekannten

- Mir selbst

- Anderen

Wem?

- Das möchte ich nicht beantworten 


\subsection{Anhang 3 (Ergebnisse der Fragebogen zum Langzeitverlauf)}

\section{Ergebnisse der Fragebogen zum Langzeitverlauf}

1. Waren Sie überhaupt jemals abhängig oder suchtkrank?

$\begin{array}{lrl}\text { Nein: } & 12 & =28 \% \\ \text { Ja: } & 25 & =58 \% \\ \text { Keine Antwort: } & 6 & =14 \% \\ \text { Insgesamt: } & 43 & =100 \%\end{array}$

Mehr als die Hälfte der Nachbefragten gab an, jemals abhängig oder suchtkrank gewesen zu sein.

2. In welchem Jahr hat Ihre Suchtkrankheit nach Ihrer Schätzung begonnen? Anzahl der Jahre von Selbsteinschätzung des Suchtbeginns bis zur Erstdiagnose:

Mit Angabe: $\quad 28$

Keine Angabe: 15

$\left.\begin{array}{rrrrr}0 & - & 4 & \text { Jahre } & 12 \\ 5 & - & 10 & \text { Jahre } & 4 \\ 11 & - & 15 & \text { Jahre } & 4 \\ 16 & - & 20 & \text { Jahre } & 3 \\ 21 & - & 30 & \text { Jahre } & 3 \\ >30 & & & \text { Jahre } & 2\end{array}\right\} \quad \begin{aligned} & 12 \\ & 16\end{aligned}$

Leider ist festzustellen, dass in den meisten Fällen der Zeitraum von der ersten Selbsteinschätzung bis zur medizinischen Erstdiagnose einen vernünftigen Zeitraum erheblich überschreitet. 
3. Welcher Suchtstoff war der erste in Ihrem Leben?

$\begin{array}{lr}\text { Benzodiazepin: } & 21 \\ \text { Andere: } & 8 \\ \text { Keine Angabe: } & 14\end{array}$

In den meisten Fällen war der erste Suchtstoff ein Benzodiazepin.

4. In welchem Jahr mussten Sie erstmals wegen Ihrer Sucht ins Krankenhaus?

Die Jahresangaben der Patienten wurden mit der Frage 2 in Beziehung gestellt. Anzahl der Jahre vom Beginn der Suchtkrankheit nach eigener Einschätzung (s. Frage 2) bis zum ersten Krankenhausaufenthalt nach eigener Einschätzung (Frage 4):

Mit Angabe: $\quad 19$

Keine Angabe: $\quad 24$

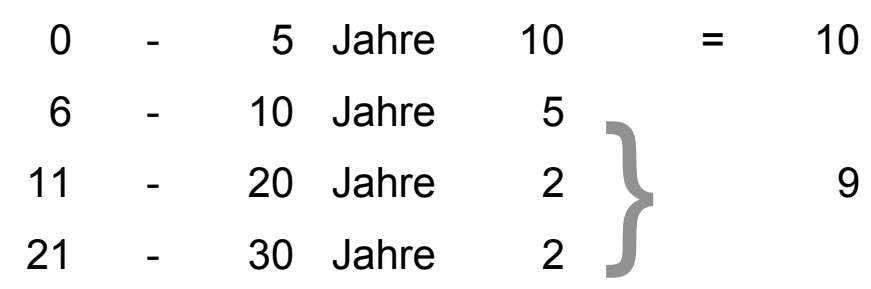

Anzahl der Jahre vom ersten Krankenhausaufenthalt nach eigener Einschätzung bis zum Erstaufenthalt in der Psychiatrie der Uniklinik Göttingen (Datum der Erstdiagnose in Patientenakte):

- 1-5 Jahre: 9

- 6-15 Jahre: 2

- Ein Patient gab an, er hätte niemals wegen einer Sucht in ein Krankenhaus gemusst (es existiert jedoch ein Erstdiagnosedatum in der Patientenakte der Psychiatrie der Uniklinik Göttingen). 
- Zwölf Patienten gaben bei der Frage, wann sie erstmals wegen ihrer Sucht ins Krankenhaus mussten, Daten an, wobei die Jahre nach einem Aufenthalt in der Psychiatrie der Uniklinik Göttingen liegen. Heißt das, dass die Patienten einen Aufenthalt in der Psychiatrie nicht als Krankenhausaufenthalt werten oder sich nicht mehr daran erinnern konnten? Oder hat dies andere Bedeutungen?

- Keine Angabe: 19

5. Wie viele stationäre und ambulante Entzüge haben Sie wegen einer Abhängigkeit gemacht?

Keine Angabe: 15

Mit Angabe: $\quad 29$ (= Entzüge insgesamt)

Keine Entzüge: $\quad 5$

Stationäre Entzüge: $\quad 18$

Ambulante Entzüge: 3

Ohne nähere Angabe, ob stationär oder ambulant: $\quad 8$

6. Wann hatten Sie Ihren ersten stationären Entzug?

Angegeben wird hier die Dauer in Jahren vom Erstdiagnosedatum in der Psychiatrie der Universität Göttingen bis zum ersten stationären Entzug:

Keine Angabe: 23

Mit Angabe: $\quad 18$

0 - 5 Jahre: 13

6 - 10 Jahre: 5

Zwei Patienten gaben ein Datum an, das vor dem Erstdiagnosedatum in der Psychiatrie der Uniklinik Göttingen lag. 
7. Wie viele Langzeittherapien haben Sie gemacht?

$\begin{array}{lrr}\text { Keine Angabe: } & 24 & \\ \text { Keine Langzeittherapie gemacht: } & 9 & \\ \text { Langzeittherapie gemacht: } & 9 \text { (teils mehrfach die Angabe von: } \\ & & \text { „fast ständig“) } \\ \text { Psychoanalysen: } & 2\end{array}$

8. Welche Suchtstoffe haben Sie jemals genommen?

Zum Teil Mehrfachnennungen:

Benzodiazepine: $\quad 28$

Andere: $\quad 6$

Keine Angabe: $\quad 16$

9. Wie war Ihre Höchstdosis (im ganzen Leben an „Benzodiazepinen = Schlaf- und Beruhigungsmitteln)?

Im Folgenden wird die genaue Auflistung der gemachten Angaben wiedergegeben:

Keine Angabe:

„Keine“:

„Keine Ahnung“: 3

"Stetig steigend": 1

„Mehrere Tbl., je nach Bedarf": 1

„Unzählbar“: 
$1 \times \operatorname{tgl}:$ : 2

$2 \times$ tgl.: 1

$3 \times \operatorname{tgl}:$ : 2

$1 \frac{1}{2}$ Tbl.: 1

2 Tbl.: $\quad 2$

4 Tbl./tgl.: $\quad 1$

5 Tbl.:

6 Tbl./tgl.: 2

10 Tbl.: 1

„180 mg Valium“: 1

„6 x 50,4 mg“:

„200 mg/Tag“: 1

„Tranxilium 203 x pro Woche“: 1

"ca. 60 mg“: 1

"40 mg/2 x Rohypnol“: 1

„12,5 mg pro Tag“: 1

"1 x 50 mg": 1

„3 x 10 mg“: $\quad 1$

Auf diese Frage wurde sehr unterschiedlich geantwortet. Die Angaben variieren zum Beispiel von „stetig steigend“, „1 x tgl.“, „mehrere Tabletten, je nach Bedarf“, „10 Tbl.“, „180 mg Valium“ bis zu „6 x 50,4 mg“ oder „unzählbar“.

Bei den meisten Angaben war zum Teil nicht festzustellen, auf welches Präparat sich diese beziehen, da viele der Befragten verschiede Schlaf- und Beruhigungsmittel zur gleichen Zeit einnahmen. 
10. Wurde Ihre Suchterkrankung durch eine andere Grundkrankheit ausgelöst (z.B. Migräne, Depression etc.)?

$\begin{array}{lr}\text { Neurologisch-psychiatrische Erkrankung: } & 23 \\ \text { Andere: } & 4 \\ \text { Nein: } & 2 \\ \text { Keine Angabe: } & 14\end{array}$

11. Wie viel rauchen Sie zurzeit täglich?

$\begin{array}{lr}\text { „Nie/Keine“: } & 10 \\ \text { „2-5 Stk./tgl.“: } & 1 \\ \text { „6 Stk./tgl.“: } & 1 \\ \text { „10 Stk./tgl.“: } & 1 \\ \text { „15-20 Stk./tgl.“: } & 3 \\ \text { „20 Stk./tgl.“: } & 4 \\ \text { „30 Stk./tgl.“: } & 1 \\ \text { „1 Packung“: } & 2 \\ \text { Keine Angabe: } & 20\end{array}$

Kommentar: Zum Zeitpunkt der Erfassung der 207 in die Studie aufgenommenen benzodiazepinabhängigen Patienten waren laut Krankenakte 41 Patienten $(19,8 \%)$ Raucher. Davon waren bereits zehn rauchende Patienten laut Akte verstorben. Somit wurden 31 Raucher angeschrieben. In den Fragebogen gaben 13 Personen an zu rauchen. 20 Patienten machten keine Angaben. Mit den vorliegenden Daten ist kein Vergleich der Angaben in den Krankenakten und den Fragebogen möglich, da die Fragebogen der noch Lebenden, darunter auch die der Raucher, nicht vollständig zurückgesandt wurden und manche der Probanden nicht angeschrieben werden konnten. 
Außerdem müssten, um exakte Daten zu erhalten und um die Dunkelziffer zu umgehen, objektive Parameter zum Nachweis eines eventuellen Nikotingebrauchs bestimmt werden (Kotinin, ein Metabolit des Nikotins und Co-Hämoglobin), doch sollte dieses Vorgehen aus forensischen Gründen auf Studien begrenzt bleiben. Ferner ist zu berücksichtigen, dass auch nicht jeder Nikotinkonsum anamnestisch erfasst wurde, da die Patienten nicht explizit danach gefragt wurden.

12. Welche Medikamente haben Sie gestern eingenommen?

Keine Angabe:

Keine:

„nur verordnete“:

„Viele: Asthma, Herz-Kreislauf, Magen“: 1

„Nexium 40 mg“:

„Anafranil 250 mg“:

“Diazepam 30, Mianserin 15, Trimineurin 50, Metoprolol, Valium 10": 1

"Biso-Puren 10 mg, Atosil 50 mg": 1

"Melleril ret. 200, Cipralex 10": 1

"Blutdruckmittel": 1

"Zucker-Tabletten": 1

"Madopar, PK-Levo, Almerid, Comtess, Trimineurin": 1

„Amiloretik, Lorzaar, Metformin 850, Tranxilium, ASS, Captohexal“: 1

„Omeprazol, Fluoxetin, Prednisolon, Prothyrid, Cimicifuga, Caltrate $600+\mathrm{D}$, Cetebe, Tebonin intens $120 \mathrm{mg}$, Metoprolol ratio 100,

Paracetamol": 1

„Voltaren Disp., Loperhoe, L-Thyroxin, Kreon“: 1

„Cassadan 3 x 0,5, Seroquel 100, $50 \mathrm{mg}$ Melperon, $100 \mathrm{mg}$

Amitryptilin, 2 x 100 mg Tiaprid, 5 mg Bisohexal, 0,4 mg Omnic“: 1

„Fluoxetin“: 1

„L-Thyroxin, 25 mg Saroten, Schmerztablette“: 1

„Dilatrend 125 mg 3 x tgl.“:

„Benalapril, Tamsulosin“: 
„1 x Truxal-Saft 80 mg“:

"Opipramol 50 mg, Depress ${ }^{1 \kappa}$ :

"Diazepam 5 mg“:

„ASS 100": 1

„Nexium 20, Sympal 25, Mirtazapin 15 ml“:

"L-Thyroxin 125“: 1

„1 1/2 Tbl. Aspirin“:

„Aponal": 1

„Seroquel 25, Mirtazapin 22,5, Lithium“: 1

"Saroten $0,751 x^{\prime \prime}:$

13. War Alkohol jemals ein Problem für Sie?

Nein: $\quad 31$

Keine Angabe: $\quad 10$

Ja: 2

Wenn ja, wie lange?

"Zeitweise“: 1

„Nur 6 Wochen, es bestand keine Sucht“: 1

14. Trinken Sie zurzeit Alkohol?

Nein: $\quad 30$

Keine Angabe: $\quad 6$

Ja: $\quad 7$

Wenn ja, wann zuletzt?

„Bei Feierlichkeiten 1-2 Gläser Wein“: 1

„Vor ein paar Tagen“: 3

"Gestern Abend": 1

„Vorgestern“: 1

"Sehr selten“:

${ }^{1}$ Ein Medikament ist unter diesem Namen nicht bekannt. 
15. Gab/gibt es Suchtkrankheiten in Ihrer Herkunftsfamilie?

Nein:

Nicht bekannt:

Keine Angabe:

Ja:

Wenn ja, welche und wer?

„Vater/Onkel alkoholabhängig“:

„Mutter tablettensüchtig“:

16. Welche Folgen Ihrer Suchtkrankheiten haben Sie (gehabt)? (Mehrfachnennungen möglich)

Keine Angabe:

Keine:

„Phobische Zustände“:

„Depression“:

„Ängste“:

„Schlafstörungen“:

„Parkinson“:

"Gewichtsabnahme“:

„Krampfanfall“:

„Gliederschmerzen“:

„Zweifel an Ärzten, die leichtsinnig mit Medikamentenverordnungen umgehen. Angst, dass für mich notwendige Medikamente durch Probieren von Hausarzt und Klinikärzten mich wieder fast ums Leben bringen“:

"Gangstörung":

„Arbeitslosigkeit, schlechte Schulleistungen“:

„Rente“:

„Unwohlsein, Schweißausbrüche, Taubheitsgefühle am ganzen Körper": 
17. Welche Entzugserscheinungen hatten Sie bisher (im ganzen Leben)? (Mehrfachnennungen möglich)

Keine Angabe:

„Ängste“:

„Zwangshandlungen“:

"Starke muskuläre Anspannung":

„Keine“:

„Unruhe, Zittern, vegetative Störungen“:

"Alle möglichen“:

„Selbstmordgedanken“:

18. Wenn Sie jetzt nüchtern (suchtstofffrei) sind, was hat Ihnen am meisten geholfen?

Keine Angabe:

"Entgiftungs-Gespräche“:

„Professor Poser":

"Soziale Kontakte“:

„Die Kraft gehabt zu haben, den Entzug durchzustehen“: 1

"Gute Psychotherapeutin“: 1

„Entzug und Wissen“:

„Der Krampfanfall mit der schweren Kopfverletzung“: 1

„Bin nicht nüchtern“:

„Selbsthilfegruppen“:

„Disziplin“:

"Suchtberatung": 1

„Mein neuer Hausarzt, der mich von den vielen Medikamenten, die ich in Ihrer Klinik bekam, innerhalb weniger Wochen mit Akupunktur befreit hat":

"Langzeittherapie“: 1

„Einsicht und Erfolgswille“:

"Musik": 1

"Meine Frau und Sport": 
19. Mein Suchtstoffproblem hat sich seit der Behandlung in der Klinik folgendermaßen entwickelt (bitte nur eine Antwort):

Seither „trocken“ und bis heute kein Rückfall:

Sehr gebessert (kleine Rückfälle, aber nicht stationär behandelt):

Gebessert (lang anhaltende Rückfälle mit stationärem Entzug, aber auch nüchterne Zeiten):

Gebessert (ich trinke wie vorher/nehme kontrolliert Tabletten):

Unverändert (ich nehme wie vorher ein):

Verschlimmert (ich brauche mehr als früher):

Ich trinke nicht mehr, nehme aber Tabletten

Welche Tabletten?: „Oxazepam, Anafranil, Aponal“:

Ich nehme keine Tabletten mehr, trinke aber:

Keine Angaben:

20. Meine Besserung verdanke ich vorwiegend (mehrere Antworten möglich):

Dem stationären Entzug: 20

Der stationären Therapie: 8

Der stationären Langzeittherapie: 1

Meinem Psychiater: 10

Der Suchtberatungsstelle: 1

Der Selbsthilfegruppe: 2

Meiner Familie/meinem Partner: 10

Freunden und Bekannten: $\quad 6$

Mir selbst: 22

Anderen: 4

Wem? „Hausarzt“, „den Schilddrüsen-

tabletten“, „Mitpatienten auf Station“

Das möchte ich nicht beantworten: 


\subsection{Anhang 4 (Ergebnisse bezüglich des Entzuges)}

\section{Ergebnisse bezüglich des Entzuges}

Von den 207 benzodiazepinabhängigen Patienten haben 137 laut Krankenakte einen Entzug durchgeführt, davon 43 mit Erfolg. Von 94 Patienten liegen keine näheren Angaben über Erfolg oder Misserfolg vor.

Keinen Entzug unternommen haben 55 Patienten, aus folgenden Gründen:

- „Vorzeitige Entlassung auf Wunsch des Patienten“

- „eine Reduktion der Dosis wurde nicht toleriert"

- "aufgrund eines zu hohen Lebensalters“

- "das Augenmerk wurde auf eine antidementielle Therapie gelegt“

- „vor Beginn des Entzuges Eintreten des Todes, meist durch Suizid“.

Von 15 Patienten liegen keine Angaben vor.

Anhand der Fragebogen haben 18 Patienten einen stationären und drei einen ambulanten Entzug mit Erfolg durchgeführt (Mehrfachnennungen wurden gemacht). Von acht Patienten sind Art und Ausgang des Entzugs unbekannt. Keinen Entzug durchgeführt haben fünf Patienten. Keine näheren Angaben liegen über 15 Patienten vor. 


\subsection{Anhang 5 (Reaktionen der Patienten auf Einwilligung / Fragebogen)}

\section{Reaktionen der Patienten auf Einverständniserklärung oder Fragebogen}

- Anruf (Gespräch mit Prof. Poser): Patientin möchte den Fragebogen nicht beantworten. Es gehe ihr gut. Hat seither keine Benzodiazepine eingenommen, ist weder depressiv noch ängstlich. War bis Pensionierung berufstätig und ist bis heute im Sportverein. Keine Depressionen. Wohlbefinden.

- E-Mail: Patientin ist überrascht, dass Daten über sie noch vorhanden sind, da sie vor über mehr als 20 Jahren nur für ein paar Tage zur Krisenintervention in der Klinik war. Ist beunruhigt, dass wir an ihre neue Adresse geraten sind und auch ihren neuen Namen kennen, da sie nur ein paar Tage in der Abteilung war und ins [...] weiterverlegt wurde, da angenommen wurde, dass sie ein hoffnungsloser Fall sei. Sie habe stationär [...] nutzen können und eine ambulante Therapie bei Herrn Prof. [...] gemacht, die sie nicht missen möchte. Sie konnte einen guten Schulabschluss machen, ein Studium und Zusatzausbildungen und habe heute auch eine Familie, über die sie sehr froh ist.

- Anruf der Ehefrau eines Patienten (Gespräch mit Prof. Poser): Ihr Ehemann sei am [...] verstorben. Die beiden letzten Jahre seines Lebens sei er „benzodiazepinfrei“ gewesen, sie selbst habe inm immer niedriger dosierte und immer weniger Tabletten gegeben. Auf diese Weise sei er um 1990 ganz frei geworden. Danach sei es ihm besser gegangen, bis er schließlich an einer Lungenembolie im $\mathrm{KH}[\ldots]$ verstorben sei. Damals sei seine Staublunge so weit fortgeschritten gewesen, dass er mehrfach nach [...] gemusst habe. Die Ehefrau gibt an, dass der Patient mit den Benzodiazepinen während einer Kur angefangen habe. Damals sei er depressiv gewesen, habe ganz gegen sein Naturell schlecht schlafen können. Er habe Schlafmittel erhalten, von denen er abhängig gewesen sei. 
- Anruf (Gespräch mit Prof. Poser): Sie nehme seit der Behandlung bei uns keine Schlaf- und Beruhigungsmittel mehr ein, ist aber noch immer depressiv. Nahm ununterbrochen Antidepressiva. Wurde von Mirtazapin sehr dick (sehr unerfreulich für sie). Nimmt derzeit Cymbalta und fühlt sich darunter sehr wohl und auch nicht dick. Sie kann sich nicht erinnern, dass sie früher einmal bei uns in Behandlung war. (Dies spricht für einen amnesiogenen Effekt der Benzodiazepine, die sie damals genommen hatte. Es ist kein Zorn der Patientin zu spüren. Den Fragebogen möchte sie nicht ausfüllen, da sie sich eben nicht daran erinnert.)

- Vermerk des Patienten auf der Einverständniserklärung: „Ich möchte an die Zeit in Ihrem Hause nicht mehr erinnert werden."

- Vermerk des Patienten auf der Einverständniserklärung: „Bin ab [...] für 3 Jahre in Spanien. Sorry.“

- Anruf (Gespräch mit Prof. Poser): Patient sei seit Entzug bei uns abstinent und es gehe ihm ausgezeichnet. Keine Ängste oder Depressionen. Er schiebt den damaligen jämmerlichen Zustand und das schwere Entzugssyndrom (Umfallen) auf die gegen seinen Willen erfolgte Gabe von Tabletten (KH [...]). Er bereut den Entzug nicht.

- Vermerk des Patienten auf dem unausgefüllten Fragebogen: Es hätte nie eine Abhängigkeit vorgelegen. (Laut Krankengeschichte liegt eine Suchterkrankung mit eindeutigen Entzugserscheinungen vor)

- Eine Ehefrau schrieb auf die Einverständniserklärung: „Mein Mann ist dazu nicht mehr in der Lage.“

- Angehöriger schrieb auf Einverständniserklärung: „Litt seit 11 Jahren an Krebs, Depressionen nicht weg bis zum Tode. Medikamentenabhängigkeit soviel besser deshalb irrelevant, Todesdatum: [...].“ 
- Angaben einer Ehefrau: „Hiermit möchte ich Ihnen mitteilen, dass mein Mann am [...] durch Selbstmord aus dem Leben geschieden ist." Telefonische Befragung durch Prof. Poser: Depression besser, aggressiv, Durchblutungsstörungen, Benzodiazepineinnahme fortgesetzt "Mein Mann war tablettensüchtig." Die Ehefrau war sehr freundlich und zeigte großes Interesse.

- Vermerk eines Patienten auf dem ausgefüllten Fragebogen: „Offensichtlich war mir nicht bekannt, durch die Einnahme von Diazepam süchtig zu werden. Deshalb habe ich mit diesem Fragebogen Probleme gehabt."

- Vermerk eines Patienten auf dem ausgefüllten Fragebogen: „Tabletten (Beruhigungs- und Schlafmittel sind für mich der gefährlichste Suchtstoff). Nach Entzug vielleicht Erkrankung an der Psyche (vorher noch latente psychische Störungen). Persönlichkeitsstörungen (ähnlich wie akute Psychosen - depressiver Art). Körperliche Störungen (Stoffwechsel) stark psychosomatisch, dauern viele Monate an. Nach heutigem Stand (Therapie, medizinisch) nicht in dem Maße heilbar wie Alkoholismus, illegale Drogensucht (meine Meinung und Erfahrung). Ich wünsche und hoffe, dass sich dies einmal ändert. PS.: Bitte entschuldigen Sie meine bewusst belehrende und provokante Art ihre Fragen zu beantworten. Meine Verbitterung ist sehr groß."

- Vermerk einer Patientin auf dem ausgefüllten Fragebogen: „Mir geht es immer noch sehr schlecht, habe in der Psychiatrie keine hilfreichen Erfahrungen gemacht sowie Fehldiagnosen. Habe vorübergehend sehr gute Erfahrungen mit einer christlichen Freikirche gemacht bzw. mit den dadurch geweckten starken Jesuserfahrungen, die aber wohl nur durch vorangehende intensive Arbeit mit alternativen Therapien (Bioenergetik, Gesprächstherapie, Gruppendynamik) möglich waren. Deutliche Verbesserung meines Befindens - dann aber leider Flucht und Abwehr. Verspreche mir im Moment am meisten Erfolg in der Umsetzung der Ratschläge in alternativen Bereichen wie oben erwähnt. Halte meine Erfahrungen auch als für Suchtkranke wesentlich. Bin esssüchtig geworden. Hatte in der psychiatrischen Beratungsstelle für Studenten (1975) eine Familientherapie begonnen, die ich zu früh mit Einverständnis des Therapeuten 
abgebrochen habe. Wäre nach meiner jetzigen Einschätzung sehr hilfreich gewesen."

- Vermerk einer Patientin auf dem ausgefüllten Fragebogen: „Habe keine Alkoholprobleme in meinem Leben je gehabt. Dieser Fragebogen passt nicht zu meiner Person, da liegt wohl ein Irrtum vor. Deshalb erhielten Sie keine Antwort von mir." (Im Fragebogen Angabe der regelmäßigen Einnahme von Aponal [Doxepin], in der Patientenakte dokumentierte Dauereinnahme von Rohypnol [Flunitrazepam] und Uskan [Oxazepam]).

- Vermerk eines Patienten auf dem nicht ausgefüllten Fragebogen: „Ich habe mit Süchten nichts zu tun. Es muss ein Irrtum sein." (Laut Krankengeschichte liegt eine Suchterkrankung vor).

- Vermerk einer Patientin auf dem ausgefüllten Fragebogen: „Keine Besserung, da ich 1986 mitten in der Behandlung entlassen wurde. Außerdem Diskrepanzen zwischen Stationsarzt und mir und eines Professors. Dieser Professor ist mir in schlechter Erinnerung. Wenn er zum Beispiel Visite auf Station machte, schimpften oder heulten mindestens $2 / 3$ der Patientinnen danach.“

- Vermerk eines Patienten auf ausgefülltem Fragebogen: „Durch den stationären Entzug war ich zwangsläufig medikamentenfrei - habe aber sofort nach Entlassung wieder Gityl 6 mg (Bromazepam) genommen für ca. 12 Monate und danach Entzug selber geschafft. Depression: vollständig verschwunden.“

- Vermerk eines Patienten auf dem nicht ausgefüllten Fragebogen: „Ich hatte massive Schlafprobleme. Mit Restex Tbl. (Levodopa + Benserazid) sind sie verschwunden. Ein Suchtproblem hatte ich nie." (Laut Krankengeschichte liegt eine Suchterkrankung vor) 
- Vermerk eines Patienten auf dem nicht ausgefüllten Fragebogen: „Da ich wegen eines Nervenzusammenbruchs, nicht aber wegen eines Suchtproblems in Ihrer Klinik behandelt wurde, trifft der mir zugesandte Fragebogen nicht (allenfalls am Rande) für mich zu. Ich habe mit 14-Stunden-Arbeitstagen (und mehr, Wochenenden eingeschlossen) in einem ca. 30-jährigen Existenzkampf gestanden. Dadurch vermochte ich kaum abzuschalten und hatte ständig Einbzw. Durchschlafprobleme. Es wurden mir unterschiedliche Schlaftabletten verschrieben, Zeit zum Ausspannen war nie gegeben. Etwa 1 1/2 Jahre nach Abgabe meines Betriebs erlitt ich einen Schlaganfall (wie sich später herausstellte, den dritten). Da mehrtägiger Dauerschlaf, wurde er vom Arzt nicht erkannt; er schickte mich im Februar 1992 zum Schlaflabor in [...]. Auch dort nicht erkannt. Gestörte Schlafarchitektur. Polysomnographie: ,während der gesamten Nacht nur 10 Minuten REM-Schlaf, keine Apnoe.' Empfohlen wurde Einnahme von jeweils 1 Tbl. Stilnox (Zolpidem). Nach kurzer Zeit konnte ich auf $1 / 2$ Tablette reduzieren, später wieder eine ganze Tablette. Häufig Hypertonie. Arztwechsel 1999. Nach vierwöchigem Schlafprotokoll stellte er Schlaf-Behandlungsbedürftigkeit fest. Diverse Schlafmittel wurden durchprobiert, schließlich mit Rohypnol (Flunitrazepam) $1 \mathrm{mg}$ zeitweise Erfolg erzielt. Geistig habe ich mich intensiv beschäftigt, Aufsätze, zwei Bücher geschrieben, kranke Frau betreut, die wegen „Pfuschoperation“ dreimal Knieprothesen bekommen musste. Das führte wiederum zu Überlastungen, wegen Herzrhythmusstörungen auch zu Panikzuständen. 1 Tbl. Stilnox führte nur noch zu Durchschlafstörungen. Nach Nervenzusammenbruch Einweisung in Ihre Klinik. Dort autogenes Training, Entspannung. Stilnox wurde durch Rohypnol ersetzt. Dadurch ungestörter Nachtschlaf. Gut erholt entlassen. Um von Schlaftabletten abzukommen, im Dezember 2004 um erneute Polysomnographie in [...] nachgesucht. Man sei auf Monate ausgebucht, eine Rohypnol $1 \mathrm{mg}$ sei zu tolerieren.

Seitdem schlafe ich mit dieser Dosis gut, ohne diese (gelegentlich weiche ich auf schwächere aus) Ein- oder Durchschlaf nicht möglich. Ob durch Schlaganfälle (insgesamt fünf) Ausstoß von Schlafhormon gestört, wurde nie untersucht. Ich nehme (außer Schlafmittel) keine Drogen, rauche nicht, bin nicht alkoholabhängig. Irgendwelche ,Suchtbehandlungen' wurden nicht durchgeführt.“ 
- Vermerk eines Patienten auf dem ausgefüllten Fragebogen: „Ich wäre nicht erkrankt, wenn ich meinem damaligen Hausarzt weniger vertraut hätte. Die Suchtgefahr hat mir vorher keiner erläutert.“

- Vermerk einer Patientin auf dem ausgefüllten Fragebogen: „Einen großen Anteil an meiner Heilung hat der damalige Oberarzt gehabt. Nach dem Aufenthalt in der Uni-Klinik hat für mich nach 20 Jahren Depression ein neues Leben begonnen."

- Vermerk einer Patientin auf dem nicht ausgefüllten Fragebogen: „Trifft für mich nicht zu!“ (Laut Patientenakte regelmäßige Einnahme von: Remestan [Temazepam], Noctamid [Lormetazepam], Staurodorm [Flurazepam] und Diagnose „Benzodiazepinabhängigkeit“.)

- Vermerk einer Patientin auf dem nicht ausgefüllten Fragebogen: „Keine Angaben. War nicht suchtkrank." (Laut Patientenakte regelmäßige Einnahme von Adumbran (Oxazepam) und Diagnose „Benzodiazepinabhängigkeit“.)

- Vermerk einer Patientin auf dem ausgefüllten Fragebogen: „Vor drei Jahren war ich in [...] in der Nervenklinik (4 Wochen lang). Ich bekam Sepram (Citalopram) verschrieben. Es half auch zunächst. In den letzten Wochen gab es Komplikationen. Nachts wachte ich von Zuckungen im Körper auf. Atemstillstand und Angstgefühle. Es war sehr schlimm! Ich dachte, es ging zu Ende. Seither nehme ich keine Tabletten mehr, nur Zuckertabletten und L-Thyroxin 100." 


\section{Literaturverzeichnis}

Allgulander C (1978): Dependence on sedative and hypnotic drugs. A comparative clinical and social study. Acta Psychiatr Scand, Supplementum $\underline{270}$

APA - American Psychiatric Association: Benzodiazepine Dependence, Toxicity, and Abuse - A Task Force Report of the American Psychiatric Association, Washington, DC 1990

APA - American Psychiatric Association: Diagnostic and Statistical Manual of Mental Disorders - DSM-IV-TR (4th edition, Text Revision). American Psychiatric Association, Washington, DC 2003. (deutsch: Saß H et al.: Diagnostisches und Statistisches Manual Psychischer Störungen- Textrevision- DSM-IV-TR. Hogrefe, Göttingen 2003)

Belleville G (2010): Mortality hazard associated with anxiolytic and hypnotic drug use in the National Population Health Survey. Can J Psychiatry $\underline{55}, 558-567$

Celano C M, Huffman J C (2011): Depression and cardiac disease: a review. Cardiol Rev $\underline{19}, 130-142$

De Gier N A, Gorgels W J, Lucassen P L, Oude Voshaar R, Zitman F (2011): Discontinuation of long-term benzodiazepine use: 10-year follow-up. Fam Pract $\underline{28}, 253-259$

De las Cuevas C, Sanz E, de la Fuente J (2003): Benzodiazepines: more "behavioural" addiction than dependence. Psychopharmacology 167, 297-303

Di Chiara G, Imperato A (1988): Drugs abused by humans preferentially increase synaptic dopamine concentrations in the mesolimbic system of freely moving rats. Proc Natl Acad Sci USA $\underline{85}, 5274-5278$ 
Essig C F (1964): Addiction to nonbarbiturate sedative and tranquilizing drugs. Clin Pharmacol Ther $\underline{5}$ : 334-343

Facklam M, Schoch P, Haefely W E (1992): Relationship between benzodiazepine receptor occupancy and potentiation of gamma-aminobutyric acid-stimulated chloride flux in vitro of four ligands of differing intrinsic efficacies. J Pharmacol Exp Ther $\underline{261}, 1106-1112$

French D D, Chirikos T N, Spehar A, Campbell R, Means H, Bulat T (2005): Effect of concomitant use of benzodiazepines and other drugs on the risk of injury in a veterans population. Drug Saf $\underline{28}$ : 1141-1150

Gerok W, Huber C, Meinertz T, Zeidler H (Hrsg.): Die Innere Medizin Referenzwerk für den Facharzt. 11. Auflage; Schattauer Verlag, Stuttgart 2007

Gibson A, Randall D, Degenhardt L (2011): The increasing mortality burden of liver disease among opioid-dependent people: cohort study. Addiction 106, 21862192

Gisev N, Hartikainen S, Chen T F, Korhonen M, Bell J S (2011): Mortality associated with benzodiazepines and benzodiazepine-related drugs among community-dwelling older people in Finland: a population-based retrospective cohort study. Can J Psychiatry $\underline{56}, 377-381$

Green T C, Grau L E, Carver H E, Kinzly M, Heimer R (2011): Epidemiologic trends and geographic patterns of fatal opioid intoxications in Connecticut, USA: 1997-2007. Drug Alcohol Depend 115, 221-228

Grimm J, Grimm W: Deutsches Wörterbuch. Verlag Zweitausendeins, in elektronischer Form erschienen als „Der digitale Grimm, Frankfurt 2004, s. u. „Sucht“, Bd. 20, Sp. 895-896 
Haefely W (1986): Biological basis of drug-induced tolerance, rebound, and dependence. Contribution of recent research on benzodiazepines. Pharmacopsychiatry $\underline{19}$, 353-61

Hausken A M, Skurtveit S, Tverdal A (2007): Use of anxiolytic or hypnotic drugs and total mortality in a general middle-aged population. Pharmacoepidemiol Drug Saf $\underline{16}, 913-918$

Hollister L E, Motzenbecker F P, Degan R O (1961): Withdrawal reactions from chlordiazepoxide ("Librium"). Psychopharmacologia 2 2, 63-68

IUPAC (International Union of Pure and Applied Chemistry), Zugänglich über die Website der IUPAC, http://www.iupac.org/

Jenkins D H (2000): Substance abuse and withdrawal in the intensive care unit. Contemporary issues. Surg Clin North Am 80, 1033-1053

Kanto J H (1985): Midazolam: the first water-soluble benzodiazepine: pharmacology, pharmacokinetics and efficacy in insomnia and anesthesia. Pharmacotherapy $\underline{5}, 138-155$

Karjalainen $\mathrm{K}$, Lintonen $\mathrm{T}$, Impinen $\mathrm{A}$, Mäkelä $\mathrm{P}$, Rahkonen $\mathrm{O}$, Lillsunde $\mathrm{P}$, Ostamo A (2010): Mortality and Causes of Death among Drugged Drivers. J Epidemiol Community Health $\underline{64}, 506-512$

Kawachi I, Colditz G A, Ascherio A, Rimm E B, Giovannucci E, Stampfer M J, Willett W C (1996): A prospective study of social networks in relation to total mortality and cardiovascular disease in men in the USA. J Epidemiol Community Health $\underline{50}, 245-51$

Keup W: Mißbrauchsmuster bei Abhängigkeit von Alkohol, Medikamenten und Drogen. Frühwarnsystem - Daten für die Bundesrepublik Deutschland 1976 1990. Lambertus, Freiburg 1993 
Koski A, Ojanperä I, Vuori E (2002): Alcohol and benzodiazepines in fatal poisonings. Alcohol Clin Exp Res 26: 956-959

Kuo C J, Liao Y T, Chen W J, Tsai S Y, Lin S K, Chen C C (2011): Causes of death of patients with methamphetamine dependence: a record-linkage study. Drug Alcohol Rev $\underline{30}, 621-628$

Lader M (1991): History of benzodiazepine dependence. J Subst Abuse Treat $\underline{8}$, 53-59

Lader M (2011): Benzodiazepines revisited - will we ever learn? Addiction 106; 2086-109

Linde O: Pharmakopsychiatrie im Wandel der Zeit. Tilia-Verlag Mensch und Medizin, Klingenmünster 1988

Meyers Konversations-Lexikon, Ein Nachschlagewerk des allgemeinen Wissens. V. Auflage, Band XVI, Bibliographisches Institut, Leipzig und Wien, 1897

Möhler H, Fritschy J M, Rudolph U (2002): A new benzodiazepine pharmacology. J Pharmacol Exp Ther $\underline{300}$, 2-8

Neutel C I, Patten S B (1997): Risk of suicide attempts after benzodiazepine and/or antidepressant use. Ann Epidemiol $\underline{7}, 568-574$

Nyhlén A, Fridell M, Hesse M, Krantz P (2011): Causes of premature mortality in Swedish drug abusers: a prospective longitudinal study 1970 - 2006. J Forensic Leg Med 18, 66-72

O’Brien Ch P (2005): Benzodiazepine Use, Abuse, and Dependence. J Clin Psychiatry $\underline{66}, 28-33$ 
Orriols L, Salmi L R, Philip P, Moore N, Delorme B, Castor A, Lagarde E (2009): The impact of medical drugs on traffic safety: a systematic review of epidemiological studies. Pharmacoepidemiol Drug Saf $\underline{18}, 647-658$

Piesiur-Strehlow B, Strehlow U, Poser W (1986): Mortality of patients dependent on benzodiazepines. Acta Psychiatr Scand $\underline{73}, 330-5$

Platz W E: Missbrauchshäufigkeit von Tranquilizern bei stationär behandelten Abhängigkeitskranken; in: Benzodiazepine - Rückblick und Ausblick; hrsg. Hippius H, Engel R R, Laakman G; Springer-Verlag, Berlin-Heidelberg-New York-Tokyo 1986, 242-252

Poser S, Eva-Condemarin P, Poser W (1997): Mortalidad y causa de muerte en adicciones. Rev Chil Neuro-Psiquiat 35, 259-265

Poser W: Langzeitverlauf und klinische Bedeutung der Benzodiazepinabhängigkeit; in: Psychiatrie für die Praxis. Benzodiazepine: Eine Standortbestimmung; hrsg. Poser W, Rüther E; MMV Medizin Verlag München 1991, 23-40

Poser W, Poser S: Medikamente - Mißbrauch und Abhängigkeit, Entstehung Verlauf - Behandlung, Georg Thieme Verlag Stuttgart - New York 1996

Poser W, Wegerer V (2001): Die isolierte Benzodiazepinabhängigkeit in der Hausarztpraxis, Suchttherapie $\underline{2}$, 204-208

Poser W, Poser S, Eva-Condemarin P (1992): Mortality in patients with dependence on prescription drugs. Drug Alcohol Depend $\underline{30}$, 49-57

Poser W, Böning J, Holzbach R, Schmidt L G: Medikamentenabhängigkeit (Sedativa, Hypnotika, Analgetika, Psychostimulanzien); in: Evidienzbasierte Suchtmedizin. Behandlungsleitlinie Substanzbezogene Störungen; hrsg. Schmidt L G, Gastpar M, Falkai P, Gaebel W; Deutscher Ärzteverlag, Köln 2006, 271-300 
Rintahaka P J, Nakagawa J A, Shewmon D A, † Kyyronen P, Shields W D (1999): Incidence of Death in Patients with Intractable Epilepsy During Nitrazepam Treatment. Epilepsia $\underline{40}, 492-496$

Roche Lexikon Medizin, s. u. Sucht, 5. Auflage; Urban u. Fischer München, 2003, S.1779

Smoller J W, Pollack M H, Wassertheil-Smoller S, Jackson R D, Oberman A, Wong N D, Sheps D (2007): Panic attacks and risk of incident cardiovascular events among postmenopausal women in the Women's Health Initiative Observational Study. Arch Gen Psychiatry $\underline{64}$, 1153-60

Sternbach L H (1971): 1,4-Benzodiazepine. Chemie und Betrachtungen zur Beziehung zwischen Struktur und Wirkung. Angewandte Chemie $\underline{83}, 70$

Sternbach L H (1983): The benzodiazepine story. J Psychoactive Drugs 15, 15-7

Strehlow U, Piesiur-Strehlow B, Poser W: Erkrankungsverlauf und Mortalität bei Suchtkranken: vorläufige Katamneseergebnisse; in: Suchtkrankheiten. Diagnose, Therapie und analytischer Nachweis; hrsg. Arnold W, Poser W E, Möller M R; Springer-Verlag, Berlin-Heidelberg-New York-London-Paris-Tokyo 1988, 236-240

Titler M G, Shever L L, Kanak M F, Picone D M, Qin R (2011): Factors associated with falls during hospitalization in an older population. Res Theory Nurs Pract $\underline{25}$, 127-148

Van't Veer-Tazelaar N, van Marwijk H, van Oppen P, Nijpels G, van Hout $H$, Cuijpers P, Stalman W, Beekman A (2006): Prevention of anxiety and depression in the age group of 75 years and over: a randomised controlled trial testing the feasibility and effectiveness of a generic stepped care programme among elderly community residents at high risk of developing anxiety and depression versus usual care [ISRCTN26474556]. BMC Public Health $\underline{18}, 186$ 
Vicens C, Socias I, Mateu C, Leiva A, Bejarano F, Sempere E, Basora E, Palop V, Mengual M, Beltran J L, Aragonès E, Lera G, Folch S, Pinol J L, Esteva M, Roca M, Arenas A, DelMar Sureda M, Campoamor F, Fiol F (2011): Comparative efficacy of two primary care interventions to assist withdrawal from long term benzodiazepine use: a protocol for a clustered, randomized clinical trial. BMC Fam Pract $\underline{12}, 12-23$

Vinkers D J, Gussekloo J, van der Mast R C, Zitman F G, Westendorp R G J (2003): Benzodiazepine Use and Risk of Mortality in Individuals Aged 85 Years or Older. JAMA J Am Med Assoc 290, 2942-2943

WHO, Weltgesundheitsorganisation (2000): Internationale Klassifikation psychischer Störungen. ICD-10 Kapitel V (F). Klinisch diagnostische Leitlinien. 4. Aufl. Hans Gruber, Bern

Winkelmayer W C, Mehta J, Wang P S (2007): Benzodiazepine use and mortality of incident dialysis patients in the United States. Kidney Int $\underline{72}, 1388-93$

Wolf B, Grohmann R, Mössner B, Rüther E, Poser W: Medikamentenmißbrauch und -abhängigkeit; in: Unerwünschte Wirkungen von Psychopharmaka Ergebnisse der AMÜP-Studie; hrsg. Grohmann R, Rüther E, Schmidt L G; Springer-Verlag, Berlin - Heidelberg - New York - London - Paris - Tokyo Hong Kong - Barcelona - Budapest 1994, 277-308

World Medical Association: Declaration of Helsinki. 5. Revision, Edinburgh 2000. Zugänglich über die Website der WMA., http://www.wma.net/e/policy/c8.htm

Ziegler G: Die Praxis der Benzodiazepinanwendung; in: Psychiatrie für die Praxis. Benzodiazepine: Eine Standortbestimmung; hrsg. Poser W, Rüther E; MMV Medizin Verlag München 1991, 41-66 
Zitman F G, Couvée J E (2011): Chronic benzodiazepine use in general practice patients with depression: an evaluation of controlled treatment and taper-off: Report on behalf of the Dutch Chronic Benzodiazepine Working Group. $\mathrm{Br} J$ Psychiatry $\underline{178}, 317-324$ 


\section{Danksagung}

Ich möchte mich herzlich bei allen Personen bedanken, die auf verschiedene Weise zum Gelingen dieser Arbeit beigetragen haben:

- Herrn Prof. Dr. med. W. Poser für die Bereitstellung des Themas und für die Betreuung während dieser Arbeit

- Herrn Prof. Dr. med. M. Oppermann für die Unterstützung zur Möglichkeit der Weiterführung und Beendigung dieser Dissertation an der Universität Göttingen

- Meinen Eltern für die Aufmunterungen in schwierigen Phasen und für das Korrekturlesen

- $\quad$ Herrn K. ed Dik für seine abschließende Korrektur und Formatierung. 